IGC-11/8-1 NSF-KITP-11-177

\title{
Superconformal symmetry and maximal supergravity in various dimensions
}

\author{
Marco Chiodaroli ${ }^{1}$, Murat Günaydin² ${ }^{2}$ and Radu Roiban ${ }^{3}$ \\ Institute for Gravitation and the Cosmos \\ The Pennsylvania State University \\ University Park PA 16802, USA \\ and \\ Kavli Institute for Theoretical Physics \\ University of California \\ Santa Barbara CA 93106, USA
}

\begin{abstract}
In this paper we explore the relation between conformal superalgebras with 64 supercharges and maximal supergravity theories in three, four and six dimensions using twistorial oscillator techniques. The massless fields of $\mathcal{N}=8$ supergravity in four dimensions were shown to fit into a CPT-self-conjugate doubleton supermultiplet of the conformal superalgebra $S U(2,2 \mid 8)$ a long time ago. We show that the fields of maximal supergravity in three dimensions can similarly be fitted into the super singleton multiplet of the conformal superalgebra $\operatorname{SP}(16 \mid 4, \mathbb{R})$, which is related to the doubleton supermultiplet of $S U(2,2 \mid 8)$ by dimensional reduction. Moreover, we construct the ultra-short supermultiplet of the six-dimensional conformal superalgebra $O S p\left(8^{*} \mid 8\right)$ and show that its component fields can be organized in an on-shell superfield. The ultra-short $O S p\left(8^{*} \mid 8\right)$ multiplet reduces to the doubleton supermultiplet of $S U(2,2 \mid 8)$ upon dimensional reduction. We discuss the possibility of a novel non-metric based $(4,0)$ six-dimensional supergravity theory with $U S p(8)$ $R$-symmetry that reduces to maximal supergravity in four dimensions and is different from six-dimensional metric based $(2,2)$ maximal supergravity, whose fields cannot be fitted into a unitary supermultiplet of a simple conformal superalgebra.

Such an interacting $(4,0)$ theory would be the non-metric gravitational analog of the interacting $(2,0)$ theory.
\end{abstract}

\footnotetext{
${ }^{1}$ email: mchiodar@gravity.psu.edu ${ }^{2}$ email: murat@phys.psu.edu ${ }^{3}$ email: radu@phys.psu.edu
} 


\section{Introduction}

Superconformal invariance and the constraints it imposes on quantum field theories have long been subject of active investigation. In two dimensions, this symmetry has been essential in the development of superstring theory. In more recent years, the invariance of four-dimensional $\mathcal{N}=4$ super Yang-Mills theory under the conformal superalgebra $P S U(2,2 \mid 4)^{1}$ has played an essential role in formulating and testing the $A d S / C F T$ correspondence $[1,2]$. From the symmetry point of view, the $A d S / C F T$ correspondence is based on the isomorphism between conformal superalgebras in $d$ spacetime dimensions and $A d S$ superalgebras in $d+1$ dimensions.

The study of Kaluza-Klein supergravity theories [3, 4, 5] has long established the critical importance of some fundamental massless conformal supermultiplets in obtaining the spectra of type $I I B$ supergravity on $A d S_{5} \times S^{5}$ and eleven-dimensional supergravity on $A d S_{7(4)} \times S^{4(7)}$. The spectrum of $I I B$ supergravity on $A d S_{5} \times S^{5}$ was obtained by tensoring the CPT-self-conjugate doubleton supermultiplet of the four-dimensional, $\mathcal{N}=$ 4 superconformal algebra $P S U(2,2 \mid 4)$ in [3], where it was pointed out that the doubleton supermultiplet does not have a Poincaré limit as a representation of the five-dimensional, $\mathcal{N}=8$ superalgebra. The authors of [3] thus suggested that the field theory of the doubleton supermultiplet lives on the boundary of $A d S_{5}$ which can be identified with Minkowski space and that the corresponding interacting theory is the $\mathcal{N}=4$ super YangMills theory which was known to be conformally invariant. Similarly, the spectra of eleven-dimensional supergravity over $A d S_{7} \times S^{4}$ and $A d S_{4} \times S^{7}$ were obtained by tensoring of doubleton and singleton supermultiplets of $O S p\left(8^{*} \mid 4\right)$ and $O S p(8 \mid 4, \mathbb{R})[4,5]$. These supermultiplets do not have a Poincaré limit as $A d S_{7}$ and $A d S_{4}$ supermultiplets and their field theories were conjectured to be conformally invariant field theories on the boundaries which are the six- and three-dimensional Minkowski spaces ${ }^{2}$. For $O S p\left(8^{*} \mid 4\right)$ with the even subgroup $S O(6,2) \times U S p(4)$ the doubleton supermultiplet is the six-dimensional, $(2,0)$ conformal supermultiplet whose interacting theory is believed to be dual to $M$-theory on $A d S_{7} \times S^{4}$. Recently it was shown that the four-dimensional, $\mathcal{N}=4$ Yang-Mills supermultiplet and the six-dimensional, $(2,0)$ conformal supermultiplet are simply the minimal unitary supermultiplets of $P S U(2,2 \mid 4)$ and $O S p\left(8^{*} \mid 4\right)$, respectively $[8,9]^{3}$, which

\footnotetext{
${ }^{1}$ By abuse of notation, we will use the same notation for supergroups and corresponding superalgebras throughout the paper.

${ }^{2}$ The singular nature of the Poincaré limit of singleton (remarkable) representations of the fourdimensional $A d S$ group discovered by Dirac [6] was shown by Fronsdal and collaborators who pointed out that their field theories live on the boundary of $A d S_{4}$ [7].

${ }^{3} \mathrm{~A}$ minimal unitary representation of a non-compact group is defined as a representation on the Hilbert space of functions of the minimal number of variables possible. The minimal unitary supermultiplets of non compact supergroups as defined and constructed in $[8,9]$ contain the minimal unitary representations and some of their deformations that are related by supersymmetry.
} 
implies that the singleton and doubleton supermultiplets are of fundamental importance, not only physically but also mathematically.

The classification of potential counterterms in $\mathcal{N}=8$ supergravity offers a novel application of the four-dimensional superconformal algebra. Based on its no-triangle behavior $[15,16,17,18,19]$, it was suggested in $[10]$ that $\mathcal{N}=8$ supergravity may be finite to all loop orders; indeed, subsequent explicit calculations show that its ultraviolet behavior matches $[11,12,13,14]$ that of $\mathcal{N}=4$ super Yang-Mills theory up to at least four loops. String dualities have also been used to argue for ultraviolet finiteness of $\mathcal{N}=8$ supergravity [20], though difficulties with decoupling towers of massive states may alter this conclusion [21]. Conservation of the Noether-Gaillard-Zumino duality current suggests $[24,25]$ another argument for finiteness; a construction of duality-invariant actions, potentially circumventing this argument, was outlined in [26]. The fields of $\mathcal{N}=8$ Poincaré supergravity can be fitted into a CPT-self-conjugate doubleton supermultiplet of the conformal superalgebra $S U(2,2 \mid 8)$ with $R$-symmetry group $U(8)[28,29]$. This superalgebra proves itself a valuable tool to study candidate counterterms which would appear at the loop order at which the theory has its first divergence. Potential on-shell counterterms should be local and should respect the on-shell symmetries of the theory. The authors of [30] have exhaustively analyzed all possible candidate counterterms identifying local supersymmetry invariants with the top components of $S U(2,2 \mid 8)$ supermultiplets that are also $S U(8)$ singlets and Lorentz scalars. Their analysis shows that any counterterm below seven loops is incompatible with the spontaneously broken $E_{7(7)}$ symmetry of the theory and recovers early results obtained though a direct construction of $E_{7(7)}$ invariants respecting linearized supersymmetry $[31,32]^{4}$.

In this paper we will show that the fields of maximal supergravity in three dimensions also fit into a unitary supermultiplet of the conformal superalgebra $O S p(16 \mid 4, \mathbb{R})$. However, a naive extension of the analysis of [30] to dimensions higher than four is hindered by the fact that, in dimensions higher than four, the maximal Poincaré supergravity multiplet is, in general, not a representation of the maximal superconformal algebra that has the full $R$-symmetry group as a factor in its even subgroup.

Interestingly, a similar issue arises considering $\mathcal{N}=4$ super Yang-Mills theory in four dimensions: while maximally supersymmetric Yang-Mills theories exist in dimensions

\footnotetext{
${ }^{4}$ An explicit potential 1/8-BPS seven-loop counterterm was recently constructed in [102]. Potential eight-loop counterterms invariant under both $E_{7(7)}$ and supersymmetry have been constructed in [32, 33]. Explicit proposals for potential linearized counterterms at three [32], five and six loops [34] have been ruled out based on their lack of $E_{7(7)}$ invariance in [35, 36] and [37, 30], respectively. The seven-loop bound on the order at which the first divergence may appear has also been obtained through string-based amplitude computations [22, 23] and through light-cone superspace arguments [27]. Together with $E_{7(7)}$ symmetry, lightcone superspace also appears to offer an argument for finiteness of $\mathcal{N}=8$ supergravity $[27]$.
} 
other than four, their supermultiplets are not representations of the corresponding superconformal algebras. In six dimensions, the theory which is constrained by $O S p\left(8^{*} \mid 4\right)$ and is the analog of four-dimensional super Yang-Mills theory is the the mysterious interacting $(2,0)$ theory discovered in $[38,39]$. Both the conformal $(2,0)$ theory and the non-conformal six-dimensional super Yang-Mills theory yield the conformal $\mathcal{N}=4$ super Yang-Mills theory when reduced to four dimensions.

In this paper, we argue that three- and four-dimensional, maximal Poincaré supergravity theories may have a similar six-dimensional counterpart whose fields at the linearized level belong to a unitary supermultiplet of six-dimensional superconformal group $O S p\left(8^{*} \mid 8\right)$. To this end, we construct the unitary irreducible representation of $O S p\left(8^{*} \mid 8\right)$ which involves only fields of spin less or equal to two and which reduces to the CPT-selfconjugate doubleton supermultiplet of $S U(2,2 \mid 8)$ in four dimensions. This supermultiplet is equivalent to the supermultiplet of $(4,0)$ linearized conformal supergravity studied earlier by Hull using the formalism of dual gravitons $[40,41]^{5}$. This multiplet may be thought of as the direct product of two $(2,0)$ multiplets, in the same sense as the four-dimensional supergravity multiplet can be interpreted as the direct product of two $\mathcal{N}=4$ Yang-Mills multiplets. The existence of this six-dimensional supermultiplet suggests the tantalizing possibility that there exists a novel interacting six-dimensional supergravity theory with maximal supersymmetry and $U S p(8) R$-symmetry, whose field content becomes, upon dimensional reduction, that of $\mathcal{N}=8$ supergravity in four dimensions, just as the fields of the six-dimensional, $(2,0)$ theory reduce to those of $\mathcal{N}=4$ super Yang-Mills in four dimensions. However, if such a $(4,0)$ interacting theory exists, it would not be a metricbased gravitational theory in the usual sense. This follows from the fact that, just like the six-dimensional Yang-Mills theory, ordinary Einstein gravity based on a metric is not conformally invariant even at the linearized level ${ }^{6}$. Just like the interacting $(2,0)$ theory we do not expect this novel interacting $(4,0)$ theory to admit a covariant action. We should stress that our main starting point, in all dimensions, is the construction of the relevant unitary representations of the conformal and superconformal groups. We show explicitly how these unitary representations are realized as covariant conformal fields and conformal supermultiplets of fields by going to the coherent state bases of the corresponding conformal groups and supergroups. Our formalism constructs directly the gauge-invariant field strengths associated with the fields of the relevant supergravity multiplets and hence guarantees that only the physical degrees of freedom appear in the representations.

In Section 2, we review the oscillator construction of the unitary representations of the four-dimensional conformal group $S U(2,2)$. In this approach, unitary irreducible representations (UIRs) of the conformal group are constructed using a set of twistorial

\footnotetext{
${ }^{5}$ We thank Henning Samtleben for bringing this work to our attention.

${ }^{6}$ Even though the interacting $(4,0)$ theory is not metric based, we shall, by an abuse of terminology, refer to it as $(4,0)$ supergravity.
} 
bosonic oscillators which obey canonical commutations relations. The conformal fields correspond to coherent states labelled by the coordinate four-vector obtained by acting with the translation generator $e^{-i x_{\mu} P^{\mu}}$ on a set of states $\left|\Phi_{j_{M}, j_{N}}^{\ell}(0)\right\rangle$ transforming covariantly in the $\left(j_{M}, j_{N}\right)$ representation of the Lorentz group $S L(2, \mathbb{C})$ with a definite scale dimension $\ell$. The states $\left|\Phi_{j_{M}, j_{N}}^{\ell}(0)\right\rangle$ can be written simply in the form $T\left|\Omega\left(j_{L}, j_{R}, E\right)\right\rangle$ where $\left|\Omega\left(j_{L}, j_{R}, E\right)\right\rangle$ are a set of states transforming irreducibly under the maximal compact subgroup $S U(2)_{L} \times S U(2)_{R} \times U(1)_{E}$ of $S U(2,2)$ such that the labels $\left(j_{L}, j_{R}, E\right)$ coincide with labels $\left(j_{M}, j_{N},-\ell\right)$ and $T$ is the intertwining operator between compact and manifestly unitary basis and the non-compact and manifestly covariant basis. The review of the oscillator construction in the compact basis is relegated to Appendix A. In Section 2.2, we discuss the extension of oscillator method to the conformal superalgebra $S U(2,2 \mid 8)$ and review the results of [28] on the construction of the CPT-self-conjugate doubleton supermultiplet of $S U(2,2 \mid 8)$. The fields of $\mathcal{N}=8$ Poincaré supergravity are in one-to-one correspondence with the fields of this CPT-self-conjugate doubleton supermultiplet. This supermultiplet has recently been used in the construction and classification of counterterms in $\mathcal{N}=8$ supergravity [30].

Section 3 is devoted to the construction of the unitary supermultiplets of the threedimensional superconformal group $O S p(16 \mid 4, \mathbb{R})$ with even subgroup $S O(16) \times S O(3,2)$. We show that the fields of maximal supergravity can be identified with the supersingleton multiplet and the four-dimensional doubleton supermultiplet of $S U(2,2 \mid 8)$ reduces to this singleton supermultiplet under dimensional reduction.

In Section 4, we review the unitary irreducible representations of the six-dimensional conformal superalgebras $O S p\left(8^{*} \mid 2 N\right)$ and construct the $(4,0)$ "chiral graviton supermultiplet" of $O S p\left(8^{*} \mid 8\right)$ with the $R$-symmetry group $U S p(8)$. In Section 4.3, using a sixdimensional superspace, we show how the fields of the $(4,0)$ supermultiplet can be organized into an on-shell superfield obeying one algebraic and one differential constraint. In Section 4.6 we write down the gauge potentials corresponding to the fields of the $(4,0)$ supermultiplet and show that they can be written as fields with mixed-symmetry Lorentz indices. We then study the dimensional reduction of six-dimensional, $(4,0)$ supermultiplet down to four dimensions. We show that the $(4,0)$ supermultiplet reduces to the CPT-self-conjugate doubleton supermultiplet of $S U(2,2 \mid 8)$.

In Section 5 we comment on the possibility of an interacting theory of the $(4,0)$ supermultiplet and on the restrictions on the allowed interactions from the higher-spin no-go theorems in the literature. We then discuss the implications of our results. 


\section{Unitary representations of $S U(2,2 \mid 8)$ and $\mathcal{N}=8$ supergravity in four dimensions}

\section{$2.1(S L(2, \mathbb{C}) \times \mathcal{D})$ covariant coherent states of $S U(2,2)$ and $4 D$ conformal fields}

The generators of the conformal group in four dimensions $S U(2,2)$ (the two sheeted covering of $S O(4,2))$ satisfy the commutation relations

$$
\left[M_{a b}, M_{c d}\right]=i\left(\eta_{b c} M_{a d}-\eta_{a c} M_{b d}-\eta_{b d} M_{a c}+\eta_{a d} M_{b c}\right) .
$$

We shall use the Minkowski metric $\eta_{a b}=\operatorname{diag}(+,-,-,-,-,+)$ with $a, b=0,1,2,3,5,6$. $M_{\mu \nu}$, with $\mu, \nu, \ldots=0,1,2,3$, are the Lorentz group generators. The four-momentum generators $P_{\mu}$, the special conformal generators $K_{\mu}$ and the dilatation generator $D$ are given by,

$$
M_{\mu 5}=\frac{1}{2}\left(P_{\mu}-K_{\mu}\right), \quad M_{\mu 6}=\frac{1}{2}\left(P_{\mu}+K_{\mu}\right), \quad M_{56}=-D .
$$

The fields of a four-dimensional conformal field theory should transform covariantly under the Lorentz group $S L(2, \mathbb{C})$ and dilatations [42]. The corresponding unitary realizations of the conformal group are induced from finite dimensional representations of the stability group $\mathcal{H}$ which is the semi-direct product of Lorentz group and dilatations with the Abelian group $\mathcal{K}_{4}$ of special conformal transformations. In other words, conformal fields live on the coset space $G / \mathcal{H}$, which in our case is just the conformal compactification of four-dimensional Minkowski spacetime. Consequently, these representations are labelled by their $S L(2, \mathbb{C})$ labels $\left(j_{M}, j_{N}\right)$, their conformal dimension $l$ and certain matrices $\kappa_{\mu}$ related to their behavior under special conformal transformations generated by $K_{\mu}$.

The stability group $\mathcal{H}$ of the origin $x^{\mu}=0$ under the action of the conformal group $S O(4,2)$ is the semi-direct product group

$$
\mathcal{H}=(S L(2, \mathbb{C}) \times \mathcal{D}) \subseteq \mathcal{K}_{4}
$$

the Lie algebra of the stability group $\mathcal{H}$ is simply the semi-direct sum of the generators $M_{\mu \nu}$ of the Lorentz group $S L(2, \mathbb{C})$, the generator $D$ of dilatations and the generators $K_{\mu}$ of special conformal transformations. We shall take complex linear combinations of the generators of the Lorentz group $S L(2, \mathbb{C})$ to represent them as $S U(2)_{M} \times S U(2)_{N}$ generators

$$
M_{m}=\frac{1}{2}\left(\frac{1}{2} \varepsilon_{m n l} M_{n l}+i M_{0 m}\right), \quad N_{m}=\frac{1}{2}\left(\frac{1}{2} \varepsilon_{m n l} M_{n l}-i M_{0 m}\right) .
$$

They satisfy

$$
\left[M_{m}, M_{n}\right]=i \varepsilon_{m n l} M_{l}, \quad\left[N_{m}, N_{n}\right]=i \varepsilon_{m n l} N_{l}, \quad\left[M_{m}, N_{n}\right]=0
$$


In Appendix A we review the oscillator construction of the positive energy unitary representations of the conformal group in a manifestly unitary compact $S U(2)_{L} \times S U(2)_{R} \times$ $U(1)_{E}$ basis. To relate the positive energy unitary representations constructed in the manifestly unitary compact basis to conformal fields transforming covariantly with respect to the Lorentz group $S L(2, \mathbb{C})$ with a definite scale dimension given by the eigenvalues of the dilatation generator $D$ we use the operator [43]

$$
T=e^{\frac{\pi}{4} M_{05}},
$$

which corresponds to a purely imaginary rotation through $\frac{\pi}{4}$. It satisfies

$$
M_{m} T=T L_{m} \quad, \quad N_{m} T=T R_{m} \quad, \quad D T=T E
$$

where $L_{m}, R_{m}$ and $E$ are the compact $S U(2)_{L} \times S U(2)_{R} \times U(1)_{E}$ generators. In other words $T$ "intertwines" the representations of the Lorentz group and dilatation generators with those of the maximal compact subgroup $S U(2)_{L} \times S U(2)_{R} \times U(1)_{E}$. Furthermore $T$ intertwines the momentum generators $P_{\mu}$ and the special conformal generators $K_{\mu}$ into operators belonging respectively to the grade +1 and the grade -1 subspaces $\mathcal{L}^{ \pm}$in the compact basis given in Appendix A. We obtain the expressions,

$$
\left(\sigma_{\mu} P^{\mu}\right)^{\alpha \beta} T=T L^{i, j}, \quad\left(\bar{\sigma}_{\mu} K^{\mu}\right)_{\alpha \beta} T=T L_{i, j} .
$$

The generators are realized in terms of oscillators obeying canonical commutation relations. The operator $T$ intertwines the oscillators that transform covariantly under the compact subgroup $S U(2)_{L} \times S U(2)_{R}$ with the oscillators that transform covariantly under the Lorentz group $S L(2, \mathbb{C})$. The oscillators $a_{i}\left(a^{i}\right)$ and $b_{i}\left(b^{i}\right)$ that transform in the $\left(\frac{1}{2}, 0\right)$ and $\left(0, \frac{1}{2}\right)$ representation of $S U(2)_{L} \times S U(2)_{R}$ go over to covariant oscillators transforming as Weyl spinors $\left(\frac{1}{2}, 0\right)$ and $\left(0, \frac{1}{2}\right)$ of the Lorentz group, respectively. Hence we shall label the covariant indices of Weyl spinors with undotted and dotted Greek indices $\alpha, \beta, \ldots \dot{\alpha}, \dot{\beta}, \ldots$,

$$
\begin{aligned}
& \eta_{\alpha}=T a_{i} T^{-1}=\frac{1}{\sqrt{2}}\left(a_{i}-b^{i}\right), \\
& \lambda^{\alpha}=T a^{i} T^{-1}=\frac{1}{\sqrt{2}}\left(a^{i}+b_{i}\right), \\
& \tilde{\eta}_{\dot{\alpha}}=T b_{i} T^{-1}=\frac{1}{\sqrt{2}}\left(b_{i}-a^{i}\right), \\
& \tilde{\lambda}^{\dot{\alpha}}=T b^{i} T^{-1}=\frac{1}{\sqrt{2}}\left(b^{i}+a_{i}\right) ;
\end{aligned}
$$

where $i, j, \ldots ; \alpha, \beta, \ldots ; \dot{\alpha}, \dot{\beta}, \ldots=1,2$ and the covariant indices on the left hand side match the indices $i, j$ on the right hand side of the equations ${ }^{7}$. They satisfy the covariant

\footnotetext{
${ }^{7}$ Here and elsewhere in this paper $\lambda$ and $\tilde{\lambda}$ will denote operators whose eigenvalues are the familiar bosonic spinors parameterizing momenta in the spinor helicity formalism. The operators $\eta$ are however unrelated to the Grassmann-odd parameters appearing in the supersymmetric extension of this formalism.
} 
commutation relations

$$
\left[\eta_{\alpha}, \lambda^{\beta}\right]=\delta_{\alpha}^{\beta}, \quad\left[\tilde{\eta}_{\dot{\alpha}}, \tilde{\lambda}^{\dot{\beta}}\right]=\delta_{\dot{\alpha}}^{\dot{\beta}}
$$

Furthermore we have

$$
\left(\sigma^{\mu} P_{\mu}\right)^{\alpha \dot{\beta}}=\lambda^{\alpha} \tilde{\lambda}^{\dot{\beta}}, \quad\left(\bar{\sigma}^{\mu} K_{\mu}\right)_{\alpha \dot{\beta}}=\eta_{\alpha} \tilde{\eta}_{\dot{\beta}}
$$

Acting with $T$ on a lowest energy irreducible representation $\left|\Omega\left(j_{L}, j_{R}, E\right)\right\rangle$ in the compact basis corresponds to a pure imaginary rotation in the corresponding representation space of $S U(2,2)$,

$$
T\left|\Omega\left(j_{L}, j_{R}, E\right)\right\rangle=e^{\frac{\pi}{4} M_{05}}\left|\Omega\left(j_{L}, j_{R}, E\right)\right\rangle
$$

Since the negative grade generators in the compact basis annihilate the lowest energy irreducible representation,

$$
\mathcal{L}^{-}\left|\Omega\left(j_{L}, j_{R}, E\right)\right\rangle=0
$$

it then follows from (2.7) that $\left|\Phi_{j_{M}, j_{N}}^{\ell}(0)\right\rangle:=T\left|\Omega\left(j_{L}, j_{R}, E\right)\right\rangle$ is an irreducible representation of the little group $\mathcal{H}$ with $S L(2, \mathbb{C})$ quantum numbers $\left(j_{M}, j_{N}\right)=\left(j_{L}, j_{R}\right)$, conformal dimension $^{8} \ell=-E$ and trivially represented special conformal transformations $K_{\mu}$ (i.e. $\left.\kappa_{\mu}=0\right)$ as a consequence of equation (2.7),

$$
K_{\mu}\left|\Phi_{j_{M}, j_{N}}^{\ell}(0)\right\rangle=0
$$

Acting with $e^{-i x^{\mu} P_{\mu}}$ on $\left|\Phi_{j_{M}, j_{N}}^{\ell}(0)\right\rangle$ one forms a coherent state labelled by the coordinate $x_{\mu}$, which we will denote as $\left|\Phi_{j_{M}, j_{N}}^{\ell}\left(x_{\mu}\right)\right\rangle$,

$$
e^{-i x^{\mu} P_{\mu}}\left|\Phi_{j_{M}, j_{N}}^{\ell}(0)\right\rangle \equiv\left|\Phi_{j_{M}, j_{N}}^{\ell}\left(x_{\mu}\right)\right\rangle
$$

These coherent states transform exactly like the states created by the action of conformal fields $\Phi_{j_{M}, j_{N}}^{\ell}\left(x_{\mu}\right)$ acting on the vacuum vector $|0\rangle$,

$$
\Phi_{j_{M}, j_{N}}^{\ell}\left(x^{\mu}\right)|0\rangle \cong\left|\Phi_{j_{M}, j_{N}}^{\ell}\left(x_{\mu}\right)\right\rangle
$$

with exact numerical coincidence of the compact and the covariant labels $\left(j_{L}, j_{R}, E\right)$ and $\left(j_{M}, j_{N},-l\right)$, respectively [43].

Let us now derive the explicit transformation properties of the coherent states under all the generators of the conformal group. First from the commutation relations of $S U(2,2)$ generators we have

$$
e^{i x \cdot P} K_{\mu} e^{-i x \cdot P}=K_{\mu}-2 x_{\mu} D+2 x^{\nu} M_{\nu \mu}+2 x_{\mu}(x \cdot P)-x^{2} P_{\mu},
$$

\footnotetext{
${ }^{8}$ In our conventions, $\ell$ is the length (or inverse mass) dimension.
} 
where $x \cdot P=x_{\mu} P^{\mu}$. This implies that

$$
\begin{aligned}
K_{\mu}\left|\Phi_{j_{M}, j_{N}}^{\ell}\left(x_{\mu}\right)\right\rangle & =e^{-i x \cdot P}\left(K_{\mu}-2 x_{\mu} D+2 x^{\nu} M_{\nu \mu}+2 x_{\mu}(x \cdot P)-x^{2} P_{\mu}\right) T\left|\Omega\left(j_{L}, j_{R}, E\right)\right\rangle \\
& =\left(-x^{2} P_{\mu}+2 x_{\mu}(x \cdot P)+2 x_{\mu} \ell+2 x^{\nu} \Sigma_{\nu \mu}\right)\left|\Phi_{j_{M}, j_{N}}^{\ell}\left(x_{\mu}\right)\right\rangle,
\end{aligned}
$$

where $\Sigma$ represents the $S L(2, \mathbb{C})$ matrices in the representation $\left(j_{M}, j_{N}\right)=\left(j_{L}, j_{R}\right)$ and $\ell$ is the conformal dimension. The last equation above can be recast in the form,

$$
K_{\mu}\left|\Phi_{j_{M}, j_{N}}^{\ell}\left(x_{\mu}\right)\right\rangle=\left(-i \frac{\partial}{\partial x^{\mu}}+2 i x_{\mu} x^{\nu} \frac{\partial}{\partial x^{\nu}}+2 \ell x_{\mu}+2 x^{\nu} \Sigma_{\nu \mu}\right)\left|\Phi_{j_{M}, j_{N}}^{\ell}\left(x_{\mu}\right)\right\rangle
$$

Similarly we have

$$
P_{\mu}\left|\Phi_{j_{M}, j_{N}}^{\ell}\left(x_{\mu}\right)\right\rangle=i \frac{\partial}{\partial x^{\mu}}\left|\Phi_{j_{M}, j_{N}}^{\ell}\left(x_{\mu}\right)\right\rangle
$$

Using

$$
D T=T E
$$

and

$$
e^{i x \cdot P} D e^{-i x \cdot P}=D-x^{\mu} P_{\mu}
$$

one finds

$$
D\left|\Phi_{j_{M}, j_{N}}^{\ell}\left(x_{\mu}\right)\right\rangle=-\left(i x^{\mu} \frac{\partial}{\partial x^{\mu}}+\ell\right)\left|\Phi_{j_{M}, j_{N}}^{\ell}\left(x_{\mu}\right)\right\rangle
$$

Using

$$
e^{i x \cdot P} M_{\mu \nu} e^{-i x \cdot P}=M_{\mu \nu}-x_{\mu} P_{\nu}+x_{\nu} P_{\mu}
$$

one finds

$$
M_{\mu \nu}\left|\Phi_{j_{M}, j_{N}}^{\ell}\left(x_{\mu}\right)\right\rangle=\left(\Sigma_{\mu \nu}-i x_{\mu} \frac{\partial}{\partial x^{\nu}}+i x_{\nu} \frac{\partial}{\partial x^{\mu}}\right)\left|\Phi_{j_{M}, j_{N}}^{\ell}\left(x_{\mu}\right)\right\rangle
$$

The lowest weight UIRs of $S U(2,2)$ with vanishing four-dimensional Poincaré mass $m^{2}=P_{\mu} P^{\mu}$ are exactly the ones obtained by taking only one generation (or color) of oscillators $\left(a_{i}\right.$ and $\left.b_{j}\right)[3,43,44]$. These are precisely the doubleton representations $[3,43,45]$. They were studied in the 1960s as "ladder representations" of the conformal group [46, 47]. More recently, it was shown that the doubleton unitary representation correspond to the minimal unitary representation of $S U(2,2)$ and one parameter deformations thereof [8]. The deformation parameter is simply the helicity of the corresponding massless conformal field.

\subsection{CPT-self-conjugate doubleton supermultiplet of $S U(2,2 \mid 8)$ and fields of $4 D, \mathcal{N}=8$ supergravity}

In this section we shall review the results of [28] where it was shown that the massless fields of $\mathcal{N}=8$ four-dimensional supergravity can be organized into the CPT-self-conjugate doubleton supermultiplet of the $\mathcal{N}=8$ superconformal algebra $S U(2,2 \mid 8)$. It is not 
clear whether this fact has direct implications for the interacting theory of this multiplet. Indeed, the corresponding supermultiplet of $\operatorname{PSU}(2,2 \mid 4)$ is the $\mathcal{N}=4$ Yang-Mills supermultiplet [3]; the interacting theory of the CPT-self-conjugate doubleton supermultiplet of $P S U(2,2 \mid 4)$ is known to be conformally invariant. However, the interacting non-linear maximal $\mathcal{N}=8$ supergravity is not conformally invariant. ${ }^{9}$

The construction of representations of the $S U(2,2 \mid 8)$ superalgebra makes use of its three graded decomposition with respect to its maximal compact subsuperalgebra $\mathcal{L}^{0}=$ $S U(2 \mid p) \times S U(2 \mid 8-p) \times U(1)$

$$
S U(2,2 \mid 8)=\mathcal{L}^{+} \oplus \mathcal{L}^{0} \oplus \mathcal{L}^{-}
$$

where

$$
\left[\mathcal{L}^{0}, \mathcal{L}^{ \pm}\right]=\mathcal{L}^{ \pm}, \quad\left[\mathcal{L}^{+}, \mathcal{L}^{-}\right]=\mathcal{L}^{0}, \quad\left[\mathcal{L}^{+}, \mathcal{L}^{+}\right]=0=\left[\mathcal{L}^{-}, \mathcal{L}^{-}\right]
$$

The Lie superalgebra $S U(2,2 \mid 8)$ can be realized in terms of bilinear combinations of bosonic and fermionic annihilation and creation operators (superoscillators) $\xi_{A}\left(\xi^{A}=\xi_{A}^{\dagger}\right)$ and $\eta_{M}\left(\eta^{M}=\eta_{M^{\dagger}}\right)$ which transform covariantly (contravariantly) under the $S U(2 \mid p)$ and $S U(2 \mid 8-p)$ sub-supergroups of $S U(2,2 \mid 8)$. We denote the annihilation and creation operators with lower and upper indices, respectively. Each superoscillator may be represented as a doublet as

$$
\xi_{A}=\left(\begin{array}{c}
a_{i} \\
\alpha_{\hat{x}}
\end{array}\right), \quad \xi^{A}=\left(\begin{array}{c}
a^{i} \\
\alpha^{\hat{x}}
\end{array}\right)
$$

and

$$
\eta_{M}=\left(\begin{array}{c}
b_{i} \\
\beta_{x}
\end{array}\right), \quad \eta^{M}=\left(\begin{array}{c}
b^{i} \\
\beta^{x}
\end{array}\right),
$$

with $i, j=1,2 ; \hat{x}, \hat{y}=1,2, . ., p ; x, y=1,2, . ., 8-p$ and

$$
\begin{aligned}
& {\left[a_{i}, a^{j}\right]=\delta_{i}^{j}, \quad\left\{\alpha_{\hat{x}}, \alpha^{\hat{y}}\right\}=\delta_{\hat{x}}^{\hat{y}},} \\
& {\left[b_{i}, b^{j}\right]=\delta_{i}^{j}, \quad\left\{\beta_{x}, \beta^{y}\right\}=\delta_{x}^{y} .}
\end{aligned}
$$

The generators of $S U(2,2 \mid 8)$ are given in terms of the above superoscillators as

$$
\mathcal{L}^{-}=\xi_{A} \eta_{M} \quad, \quad \mathcal{L}^{0}=\xi^{A} \xi_{B} \oplus \eta^{M} \eta_{N} \quad, \quad \mathcal{L}^{+}=\xi^{A} \eta^{M}
$$

The $S U(8)$ generators, written in terms of fermionic oscillators $\alpha$ and $\beta$, read as follows,

$$
A_{\hat{x}}^{\hat{y}}=\alpha^{\hat{y}} \alpha_{\hat{x}}-\frac{1}{p} \delta_{\hat{x}}^{\hat{y}} N_{\alpha}
$$

\footnotetext{
${ }^{9} \mathrm{An}$ interesting possibility is that the violation if scale invariance occurs only at the classical level; a consequence of such a scenario would be that the theory is finite.
} 


$$
\begin{aligned}
B_{x}^{y} & =\beta^{y} \beta_{x}-\frac{1}{q} \delta_{x}^{y} N_{\beta}, \\
C & =-\frac{1}{p} N_{\alpha}-\frac{1}{(8-p)} N_{\beta}+1, \\
L_{\hat{x} x} & =\alpha_{\hat{x}} \beta_{x}, \\
L^{x \hat{x}} & =\beta^{x} \alpha^{\hat{x}},
\end{aligned}
$$

where $N_{\alpha}=\alpha^{\hat{y}} \alpha_{\hat{y}}$ and $N_{\beta}=\beta^{x} \beta_{x}$ are the fermionic number operators.

Similarly, the $S U(2,2)$ generators, written in terms of bosonic oscillators $a$ and $b$, read

$$
\begin{aligned}
L_{i j} & =a_{i} b_{j}, \\
L^{i j} & =a^{i} b^{j}, \\
L_{j}^{i} & =a^{i} a_{j}-\frac{1}{m} \delta_{i}^{j} N_{a}, \\
R_{j}^{i} & =b^{i} b_{j}-\frac{1}{n} \delta_{j}^{i} N_{b}, \\
E & =\frac{1}{2} N_{a}+\frac{1}{2} N_{b}+1,
\end{aligned}
$$

where $N_{a} \equiv a^{i} a_{i}, N_{b} \equiv b^{i} b_{i}$ are the bosonic number operators.

Starting from a set of vectors $|\Omega\rangle$ in the Fock space transforming irreducibly under $S U(2 \mid 4) \times S U(2 \mid 4) \times U(1)$ and annihilated by $\mathcal{L}^{-}$, one can generate the doubleton UIRs of $S U(2,2 \mid 8)$ by repeated application of $\mathcal{L}^{+}$

$$
|\Omega\rangle, \quad \mathcal{L}^{+1}|\Omega\rangle, \quad \mathcal{L}^{+1} \mathcal{L}^{+1}|\Omega\rangle, \ldots
$$

The irreducibility of doubleton UIRs of $S U(2,2 \mid 8)$ as constructed is a consequence of the irreducibility of $|\Omega\rangle$ under $S U(2 \mid 4) \times S U(2 \mid 4) \times U(1)$.

In the subspace involving purely bosonic oscillators one gets the subalgebra $S U(2,2)$ and the above construction yields its positive energy doubleton UIRs. In the subspace involving purely fermionic oscillators one gets the compact $R$-symmetry group $S U(8)$ and the resulting representations of $S U(8)$ in its $S U(4) \times S U(4) \times U(1)$ basis.

The positive energy UIRs of $S U(2,2 \mid 8)$ decompose into a direct sum of finitely many positive energy UIRs of $S U(2,2)$ transforming in definite irreducible representations of the $R$-symmetry group $S U(8)$. Thus, each positive energy UIR of $S U(2,2 \mid 8)$ corresponds to a supermultiplet of four-dimensional massless fields.

The CPT-self-conjugate supermultiplet of $S U(2,2 \mid 8)$ is obtained by choosing the Fock vacuum as the lowest weight vector $|\Omega\rangle=|0\rangle$ in the $S U(2 \mid 4) \times S U(2 \mid 4) \times U(1)$ basis; it was given in [28] and we reproduce it in Table 1.

On-shell fields of linearized $\mathcal{N}=8$ supergravity in four dimensions satisfy massless free field equations that are conformally invariant. The nonlinear completion of the $\mathcal{N}=8$ supergravity breaks the conformal supersymmetry algebra $S U(2,2 \mid 8)$ to $\mathcal{N}=8$ Poincaré supersymmetry. There is no known conformal supergravity with the same field content 


\begin{tabular}{|c|c|c|c|c|c|}
\hline Lowest weight vector & $S U(2)_{j_{1}} \times S U(2)_{j_{2}}$ & $E_{0}$ & $S U(8)$ & $U(1)_{A}$ & Fields \\
\hline$|0\rangle$ & $(0,0)$ & 1 & $\mathbf{7 0}$ & 0 & $\phi^{[A B C D]}$ \\
\hline$L^{i x}|0\rangle$ & $\left(\frac{1}{2}, 0\right)$ & $\frac{3}{2}$ & $\mathbf{5 6}$ & 1 & $\lambda_{+}^{[A B C]} \equiv \lambda_{\alpha}^{[A B C]}$ \\
\hline$L^{i \hat{x}}|0\rangle$ & $\left(0, \frac{1}{2}\right)$ & $\frac{3}{2}$ & $\overline{\mathbf{5 6}}$ & -1 & $\lambda_{-[A B C]} \equiv \lambda_{\dot{\alpha}[A B C]}$ \\
\hline$\left(L^{i x}\right)^{2}|0\rangle$ & $(1,0)$ & 2 & $\mathbf{2 8}$ & 2 & $h_{\mu \nu}^{+[A B]} \equiv h_{(\alpha \beta)}^{[A B]}$ \\
\hline$\left(L^{i \hat{x}}\right)^{2}|0\rangle$ & $(0,1)$ & 2 & $\overline{\mathbf{2 8}}$ & -2 & $h_{\mu \nu[A B]}^{-} \equiv h_{(\dot{\alpha} \dot{\beta})[A B]}$ \\
\hline$\left(L^{i x}\right)^{3}|0\rangle$ & $\left(\frac{3}{2}, 0\right)$ & $\frac{5}{2}$ & $\mathbf{8}$ & 3 & $\partial_{[\mu} \psi_{\nu]}^{+A} \equiv \psi_{(\alpha \beta \gamma)}^{A}$ \\
\hline$\left(L^{i \hat{x}}\right)^{3}|0\rangle$ & $\left(0, \frac{3}{2}\right)$ & $\frac{5}{2}$ & $\overline{\mathbf{8}}$ & -3 & $\partial_{[\mu} \psi_{\nu] A}^{-} \equiv \psi_{(\dot{\alpha} \dot{\beta} \dot{\gamma}) A}$ \\
\hline$\left(L^{i x}\right)^{4}|0\rangle$ & $(2,0)$ & 3 & 1 & 4 & $R_{(\alpha \beta \gamma \gamma)}$ \\
\hline$\left(L^{i \hat{x}}\right)^{4}|0\rangle$ & $(0,2)$ & 3 & 1 & -4 & $R_{(\dot{\alpha} \dot{\gamma} \dot{\gamma} \dot{\delta})}$ \\
\hline
\end{tabular}

Table 1: The CPT-self-conjugate doubleton supermultiplet of $S U(2,2 \mid 8)$ defined by the lowest weight vector $|0\rangle$ in the $S U(2 \mid 4) \times S U(2 \mid 4) \times U(1)$ basis. The first column indicates the lowest weight vectors of $S U(2,2) \times S U(8)$. The oscillator formalism gives directly the gauge-invariant fields strengths associated with the fields in the representation. $A, B, C, . .=1,2, . ., 8$ are the $\mathrm{SU}(8) R$-symmetry indices. The indices $\alpha, \beta, .$. and $\dot{\alpha}, \dot{\beta}, .$. denote the chiral and anti-chiral spinorial indices of $S L(2, \mathbb{C})$. Round (square) brackets indicate symmetrization (antisymmetrization) of the enclosed indices.

as maximal supergravity in four dimensions. The superalgebra $S U(2,2 \mid 8)$ and the supermultiplet of fields given in Table 1 have been used in the construction and analysis of potential higher-loop counterterms to maximal supergravity [29, 30].

\subsection{Four-dimensional constrained superfields}

It has long been known that fields of the CPT-self-conjugate doubleton supermultiplet of $S U(2,2 \mid 8)$ can be assembled into a scalar superfield $W_{a b c d}[48,49,50]$ which depends on the $\mathcal{N}=8$ superspace coordinates,

$$
\left(x^{\alpha \dot{\beta}}, \theta^{\alpha a}, \bar{\theta}_{b}^{\dot{\beta}}\right)
$$

with $\alpha, \dot{\beta}=1,2$ and $a=1,2, \ldots 8$. One can introduce superspace covariant derivatives obeying the relation

$$
\left\{D_{\alpha a}, \bar{D}_{\dot{\beta}}^{b}\right\}=2 i \delta_{a}^{b} \partial_{\alpha \dot{\beta}}
$$

The superfield $W_{a b c d}$ transforms in the $\mathbf{7 0}$ representation of $S U(8)$ and obeys the selfduality condition

$$
\bar{W}^{a b c d}=\frac{1}{4 !} \epsilon^{a b c d e f g h} W_{e f g h}
$$


and the differential constraint

$$
\bar{D}_{\dot{\alpha}}^{a} W_{b c d e}+\frac{4}{5} \delta_{[b}^{a} \bar{D}_{\dot{\alpha}}^{f} W_{c d e] f}=0
$$

The $\theta^{0}$ component of the superfield gives the 70 scalar fields of the $\mathcal{N}=8$ supergravity multiplet. The differential constraint ensures that the components with one or more fermionic coordinates give the fields listed in Table 1 without any extra degrees of freedom. Closure of the supersymmetry algebra requires that all fields in the expansion of $W_{a b c d}$ obey free-field equations of motion.

In the following sections we will introduce analogous constrained superfields for the fields of maximal supergravity in three and six dimensions.

\section{Unitary representations of $O S p(16 \mid 4, \mathbb{R})$ and $\mathcal{N}=16$ super- gravity in three dimensions}

\subsection{Coherent states of the positive energy unitary representations of $S p(4, \mathbb{R})$ and conformal fields in three dimensions}

As illustrated in detail in Section 2, conformal fields in $d$ dimensions correspond to coherent states labeled by the coordinate $d$-vector. They are of the form

$$
e^{-i x_{\mu} P^{\mu}} T|\Omega\rangle
$$

where $T$ is the intertwiner to the non-compact basis and $|\Omega\rangle$ is the lowest energy irreducible representation of the maximal compact subgroup $S O(d) \times S O(2)$ of the conformal group $S O(d, 2)$. The lowest energy irreducible representation $|\Omega\rangle$ is obtained by the action of creation operators on the Fock vacuum $|0\rangle$ in the compact (unitary) basis. The intertwiner $T$ can be used to convert the oscillators in the compact basis to covariant oscillators in the non-compact basis acting on the "covariant Fock vacuum" $T|\Omega\rangle$. The coherent state basis makes the analysis of dimensional reduction of a conformal field or of a supermultiplet of fields very simple both in the compact unitary basis or in the non-compact covariant basis.

For example in the non-compact picture consider the above coherent state at the origin $x_{\mu}=0$, which is simply $T|\Omega\rangle$ which transform irreducibly under the Lorentz group $S O(d-1,1)$ with a definite scale dimension $\ell$. One decomposes this irreducible representation with respect to the Lorentz group of the lower dimension and restrict the coordinate vector $x_{\mu}$ to lie in the lower dimensional Minkowski subspace. Since the entire coordinate dependence comes from the action of translation generator $e^{-i x_{\mu} P^{\mu}}$ on $T|\Omega\rangle$ this decomposition yields directly the fields in the lower dimension. The only subtlety comes in 
identifying the states that correspond to derivatives (descendants) of (primary) conformal fields in the lower dimension. This identification is equivalent to dualization of the fields in the lower dimension modulo the caveat that the oscillator method always yields states corresponding to gauge invariant field strengths as opposed to gauge potentials.

\section{2 $S O(3,2) \approx S p(4, \mathbb{R})$ representations via the oscillator method}

The conformal group of three-dimensional Minkowskian spacetime is $S O(3,2)$ whose covering group is $S p(4, \mathbb{R})$. The compact three-grading of the Lie algebra of $S p(4, \mathbb{R}) \approx$ $S O(3,2)$ is determined by its maximal compact subalgebra $S U(2) \oplus U(1)$,

$$
S O(3,2)=A_{i j} \oplus U_{j}^{i} \oplus A^{i j}
$$

where $U_{j}^{i}$ denotes the $S U(2) \times U(1)$ generators. The non-compact three-grading is determined by the dilatation generator $\mathcal{D}$

$$
S O(3,2)=P_{\mu} \oplus\left(M_{\nu}^{\mu}+\mathcal{D}\right) \oplus K_{\mu}
$$

where $\mu, \nu=0,1,2$ and $P_{\mu}, M_{\nu}^{\mu}$ and $K_{\mu}$ denote translation, Lorentz and special conformal generators, respectively.

To construct the positive-energy UIRs of $S p(4, \mathbb{R})$, one introduces an arbitrary number of twistorial bosonic oscillators. However, unlike the case of $S U(2,2)$ or of $S O^{*}(8)$, where one has to choose an even number of oscillators $a_{i}(1), \ldots, a_{i}(P) ; b_{i}(1), \ldots, b_{i}(P)$ to realize the Lie algebra, here one also has the freedom of choosing an odd number. In particular one can realize the Lie algebra of $S p(4, \mathbb{R})$ in terms of a single set of twistorial bosonic oscillators, which lead to the famous singleton representations of Dirac [6].

Let $a_{i}(R), b_{i}(R)$ be a set of bosonic annihilation operators with their hermitian conjugate creation operators $a^{i}(R), b^{i}(R)$, which transform covariantly and contravariantly, respectively, under $S U(2)$. Let $i=1,2$ and $R=1, \ldots, P$ where $P$ is the number of colors or generations of oscillators. In addition, let $c_{i}$ and its conjugate $c^{i}$ be a single set of such oscillators. They satisfy the commutation relations,

$$
\left[a_{i}(R), a^{j}(S)\right]=\delta_{i}^{j} \delta_{R S}, \quad\left[b_{i}(R), b^{j}(S)\right]=\delta_{i}^{j} \delta_{R S}, \quad\left[c_{i}, c^{j}\right]=\delta_{i}^{j} \quad,
$$

while all the other commutators vanish. The vacuum state $|0\rangle$ is annihilated by all $a_{i}(R)$ and $b_{i}(R)$ as well as by all $c_{i}$.

The singleton realization of $S p(4, \mathbb{R})$ is given by the following bilinears of $c_{i}$ and $c^{i}$,

$$
M_{j}^{i}=\frac{1}{2}\left(c^{i} c_{j}+c_{j} c^{i}\right) \quad, \quad A_{i j}=c_{i} c_{j} \quad, \quad A^{i j}=c^{i} c^{j}
$$

For the above realization of $S p(4, \mathbb{R})$, there exist only two lowest energy irreducible representations of $S U(2)$ that are annihilated by $A_{i j}$. They are the Fock vacuum $|0\rangle$ and the 
one particle excitation $a^{i}|0\rangle$. These lowest energy irreducible representations determine the scalar and spinor singleton representations of $S p(4, \mathbb{R})$. The corresponding coherent states

$$
e^{i P_{\mu} x^{\mu}} T|0\rangle \equiv|\phi(x)\rangle
$$

and

$$
e^{i P_{\mu} x^{\mu}} T c^{i}|0\rangle \equiv\left|\psi^{\alpha}(x)\right\rangle
$$

describe massless scalar and spinor conformal fields in three dimensions. The intertwiner operator $T$ converts oscillators transforming covariantly under the $S U(2)$ subgroup of $S p(4, \mathbb{R})$ into oscillators transforming covariantly with respect to the Lorentz group $S U(1,1)$,

$$
\lambda^{\alpha}=T c^{i} T^{-1}, \quad \mu_{\alpha}=T c_{i} T^{-1}
$$

where $\alpha, \beta=1,2$. The masslessness follows from the fact that the Poincaré mass operator $P_{\mu} P^{\mu}$ vanishes identically for the singleton irreducible representations. When restricted to the Poincaré subgroup these representations coincide with the two massless representations of Poincaré group in three dimensions that were classified in [44]. They are the only massless representations of the Poincaré group in three dimensions.

General positive energy unitary representations of the three-dimensional conformal group are obtained by realizing its Lie algebra as bilinears of an arbitrary set of twistorial bosonic oscillators in the following manner,

$$
\begin{aligned}
U_{j}^{i} & =\mathbf{a}^{i} \cdot \mathbf{a}_{j}+\mathbf{b}_{j} \cdot \mathbf{b}^{i}+\frac{\epsilon}{2}\left(c^{i} c_{j}+c_{j} c^{i}\right), \\
A_{i j} & =\mathbf{a}_{i} \cdot \mathbf{b}_{j}+\mathbf{a}_{j} \cdot \mathbf{b}_{i}+\epsilon c_{i} c_{j}, \\
A^{i j} & =\mathbf{a}^{i} \cdot \mathbf{b}^{j}+\mathbf{a}^{j} \cdot \mathbf{b}^{i}+\epsilon c^{i} c^{j},
\end{aligned}
$$

where $\epsilon=0(\epsilon=1)$ if the number of oscillators $n$ is even (odd) and the dot product denotes summation over the generation or color index. The generators satisfy $[5,51]$

$$
\left[A_{i j}, A^{k l}\right]=\delta_{i}^{k} U_{j}^{l}+\delta_{i}^{l} U^{k}{ }_{j}+\delta_{j}^{k} U_{i}^{l}+\delta_{j}^{l} U_{i}^{k}
$$

$U^{i}{ }_{j}$ form the maximal compact subalgebra $U(2)$ of $S p(4, \mathbb{R})$. The $U(1)$ charge which defines the compact three-grading is given by the trace $U^{i}{ }_{i}$,

$$
E=\frac{1}{2} U^{i}{ }_{i}=\frac{1}{2} N_{B}+P+\frac{\epsilon}{2}
$$

and corresponds to the conformal Hamiltonian. The positive energy UIRs of $S p(4, \mathbb{R})$ can be constructed by choosing a set of states $|\Omega\rangle$ that transforms irreducibly under $U(2)$ and is annihilated by all the grade -1 generators. Repeated action on these $|\Omega\rangle$ by the grade +1 generators gives the "particle basis" of the positive energy UIRs. Poincaré 
mass does not vanish for the positive energy unitary representations of $S p(4, \mathbb{R})$ when the number $(2 P+\epsilon)>1$. Hence, the conformal fields defined by the corresponding coherent states are not massless; this is consistent with the fact that there exist only two massless representations of the Poincaré group in $d=3$.

\section{3 $S O(16)$ representations via the oscillator method}

$S O(16)$ has a three-grading structure with respect to its subgroup $U(8)$. To realize its Lie algebra we consider fermionic annihilation and creation operators that transform as $\overline{8}$ and $\mathbf{8}$ representations of $U(8)$ satisfying the canonical anti-commutation relations,

$$
\left\{\alpha_{\kappa}(R), \alpha^{\rho}(S)\right\}=\delta_{\kappa}^{\rho} \delta_{R S}, \quad\left\{\beta_{\kappa}(R), \beta^{\rho}(S)\right\}=\delta_{\kappa}^{\rho} \delta_{R S}, \quad\left\{\gamma_{\kappa}, \gamma^{\rho}\right\}=\delta_{\kappa}^{\rho} \quad \text { (if present) }
$$

where $R=1, \ldots, P$ and $\kappa, \rho=1, \ldots, 8$. Once again, the vacuum state $|0\rangle$ is annihilated by all the annihilation operators $\alpha_{\kappa}(R), \beta_{\kappa}(R)$ and $\gamma_{\kappa}$ (if present) for all values of $\kappa$ and $R$. The singletonic realization of $S O(16)$ is achieved in terms of a single set of oscillators,

$$
M_{\rho}^{\kappa}=\frac{1}{2}\left(\gamma^{\kappa} \gamma_{\rho}-\gamma_{\rho} \gamma^{\kappa}\right) \quad, \quad A_{\kappa \rho}=\gamma_{\kappa} \gamma_{\rho} \quad, \quad A^{\kappa \rho}=\gamma^{\kappa} \gamma^{\rho}
$$

where $M_{\rho}^{\kappa}$ are the generators of the $U(8)$ subgroup. This realization leads to two irre-

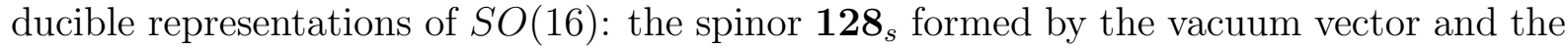

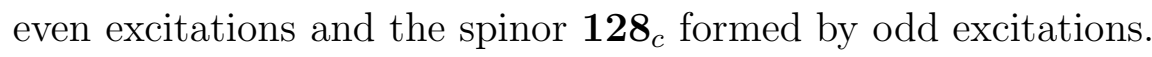

The general realization of Lie algebra of $S O(16)$ as bilinears of the above fermionic oscillators is as follows,

$$
\begin{aligned}
M_{\rho}^{\kappa} & =\boldsymbol{\alpha}^{\kappa} \cdot \boldsymbol{\alpha}_{\rho}-\boldsymbol{\beta}_{\rho} \cdot \boldsymbol{\beta}^{\kappa}+\frac{\epsilon}{2}\left(\gamma^{\kappa} \gamma_{\rho}-\gamma_{\rho} \gamma^{\kappa}\right), \\
A_{\kappa \rho} & =\boldsymbol{\alpha}_{\kappa} \cdot \boldsymbol{\beta}_{\rho}-\boldsymbol{\alpha}_{\rho} \cdot \boldsymbol{\beta}_{\kappa}+\epsilon \gamma_{\kappa} \gamma_{\rho}, \\
A^{\kappa \rho} & =\boldsymbol{\alpha}^{\kappa} \cdot \boldsymbol{\beta}^{\rho}-\boldsymbol{\alpha}^{\rho} \cdot \boldsymbol{\beta}^{\kappa}+\epsilon \gamma^{\kappa} \gamma^{\rho} .
\end{aligned}
$$

where $\epsilon=0$ or $\epsilon=1$ depending on whether we have an even or odd number of oscillators. The generators satisfy

$$
\left[A_{\kappa \rho}, A^{\lambda \sigma}\right]=\delta_{\kappa}^{\lambda} M_{\rho}^{\sigma}-\delta_{\kappa}^{\sigma} M_{\rho}^{\lambda}-\delta_{\rho}^{\lambda} M_{\kappa}^{\sigma}+\delta_{\rho}^{\sigma} M_{\kappa}^{\lambda}
$$

The $U(1)$ charge that determines the three-grading is given by

$$
C=\frac{1}{2} M_{\kappa}^{\kappa}=\frac{1}{2} N_{F}-2 P-\epsilon .
$$

The set of vectors $|\Omega\rangle$ that transform irreducibly under $U(8)$ and are annihilated by $A_{\kappa \rho}$ lead to irreducible representations of $S O(16)$ by the action of the grade +1 generators $A^{\kappa \rho}$. 


\subsection{Unitary representations of $O S p(16 \mid 4, \mathbb{R})$ via the oscillator method}

The superalgebra $O S p(16 \mid 4, \mathbb{R})$ has a three-grading with respect to its compact subsuperalgebra $U(2 \mid 8)$. To construct the positive energy UIRs of $O S p(16 \mid 4, \mathbb{R})$, consider the $U(2 \mid 8)$-covariant super-oscillators defined as follows,

$$
\begin{aligned}
\xi_{A}(R) & =\left(\begin{array}{c}
a_{i}(R) \\
\alpha_{\kappa}(R)
\end{array}\right), \quad \xi^{A}(R)=\xi_{A}(R)^{\dagger}=\left(\begin{array}{c}
a^{i}(R) \\
\alpha^{\kappa}(R)
\end{array}\right), \\
\eta_{A}(R) & =\left(\begin{array}{c}
b_{i}(R) \\
\beta_{\kappa}(R)
\end{array}\right), \quad \eta^{A}(R)=\eta_{A}(R)^{\dagger}=\left(\begin{array}{c}
b^{i}(R) \\
\beta^{\kappa}(R),
\end{array}\right) \\
\zeta_{A} & =\left(\begin{array}{c}
c_{i} \\
\gamma_{\kappa}
\end{array}\right), \quad \zeta^{A}=\zeta_{A}^{\dagger}=\left(\begin{array}{c}
c^{i} \\
\gamma^{\kappa}
\end{array}\right) .
\end{aligned}
$$

where $i=1,2, \kappa=1, \ldots, 8$ and $R=1, \ldots, P$. The oscillators $a, b, \alpha$ and $\beta$ as well as $c$ and $\gamma$ satisfy the usual (anti)commutation relations discussed in the previous subsections; it is easy to see that they imply that

$$
\left[\xi_{A}(R), \xi^{B}(S)\right\}=\delta_{A}^{B} \delta_{R S}, \quad\left[\eta_{A}(R), \eta^{B}(S)\right\}=\delta_{A}^{B} \delta_{R S}, \quad\left[\zeta_{A}, \zeta^{B}\right\}=\delta_{A}^{B} .
$$

The Lie superalgebra $O S p(16 \mid 4, \mathbb{R})$ can then be realized as follows:

$$
\begin{aligned}
M_{B}^{A} & =\boldsymbol{\xi}^{A} \cdot \boldsymbol{\xi}_{B}+(-1)^{(\operatorname{deg} A)(\operatorname{deg} B)} \boldsymbol{\eta}_{B} \cdot \boldsymbol{\eta}^{A}+\frac{\epsilon}{2}\left(\zeta^{A} \zeta_{B}+(-1)^{(\operatorname{deg} A)(\operatorname{deg} B)} \zeta_{B} \zeta^{A}\right) \\
A_{A B} & =\boldsymbol{\xi}_{A} \cdot \boldsymbol{\eta}_{B}+\boldsymbol{\eta}_{A} \cdot \boldsymbol{\xi}_{B}+\epsilon \zeta_{A} \zeta_{B} \\
A^{A B} & =\boldsymbol{\eta}^{B} \cdot \boldsymbol{\xi}^{A}+\boldsymbol{\xi}^{B} \cdot \boldsymbol{\eta}^{A}+\epsilon \zeta^{B} \zeta^{A} .
\end{aligned}
$$

$M_{B}^{A}$ generate the subalgebra $U(2 \mid 8)$ and $A_{A B}$ and $A^{A B}$ extend it to the full $O S p(16 \mid 4, \mathbb{R})$ superalgebra. The Abelian $U(1)$ charge which defines the above three-grading is

$$
E+C=\frac{1}{2} M_{A}^{A}=\frac{1}{2}\left(N_{B}+N_{F}\right)-P-\frac{\epsilon}{2} .
$$

Given this super-oscillator realization, the construction of positive energy UIRs of $O S p(16 \mid 4, \mathbb{R})$ proceeds by first choosing a set of states $|\Omega\rangle$ in the Fock space that transforms irreducibly under $U(2 \mid 8)$ and is annihilated by the grade -1 generators and then

acting on it with the grade +1 generators. This generates an infinite set of states that form the particle basis of a positive energy UIR of $O S p(16 \mid 4, \mathbb{R})$.

\subsection{Supersingletons of $O S p(16 \mid 4, \mathbb{R})$ and fields of maximal supergravity in three dimensions}

When one reduces maximal supergravity to three dimensions, all the dynamical bosonic and fermionic fields can be dualized to scalar and spinor fields and the resulting theory can 
be written as an $\mathcal{N}=16$ supersymmetric sigma model $[52,53]$ with the scalar manifold

$$
\mathcal{M}_{3}=\frac{E_{8(8)}}{S O(16)} .
$$

The scalar and spinor fields transform irreducibly as $\mathbf{1 2 8}_{s}$ and $\mathbf{1 2 8}_{c}$ of $S O(16)$, respectively. As in four dimensions one can fit the 128 massless scalar fields and the 128 massless spinor fields into a conformal supermultiplet with $R$-symmetry $S O(16)$. This supermultiplet is the supersingleton of $\operatorname{OSp}(16 \mid 4, \mathbb{R})$ obtained by considering a single set of superoscillators (i.e. the realization (3.19) for $P=0$ and $\epsilon=1$ ),

$$
M_{B}^{A}=\frac{1}{2}\left(\zeta^{A} \zeta_{B}+(-1)^{(\operatorname{deg} A)(\operatorname{deg} B)} \zeta_{B} \zeta^{A}\right) \quad, \quad A_{A B}=\zeta_{A} \zeta_{B} \quad, \quad A^{A B}=\zeta^{B} \zeta^{A} .
$$

In this case there exist two possible lowest energy irreducible representations of $U(2 \mid 8)$ annihilated by $A_{A B}$, namely the Fock vacuum $|0\rangle$ and the one superparticle state $\zeta^{A}|0\rangle$. Fock vacuum leads to a supermultiplet which in the coherent state basis can be decomposed as

$$
\mathbf{1 2 8}_{s}|\phi(x)\rangle \oplus \mathbf{1 2 8}_{c}\left|\psi^{\alpha}(x)\right\rangle .
$$

The $U(2 \mid 8)$ irreducible representation $\zeta^{A}|0\rangle$ leads to the supermultiplet

$$
\mathbf{1 2 8}_{c}|\phi(x)\rangle \oplus \mathbf{1 2 8}_{s}\left|\psi^{\alpha}(x)\right\rangle .
$$

Thus the fields of maximal Poincaré supergravity can be identified with the supersingleton multiplet of $\mathcal{N}=16$ conformal superalgebra $\operatorname{SSp}(16 \mid 4, \mathbb{R})$ defined by the vacuum vector as the lowest weight state.

Let us now show that the doubleton supermultiplet of $S U(2,2 \mid 8)$ corresponding to the fields of $d=4$ maximal supergravity reduces to the supersingleton multiplet of $O S p(16 \mid 4, \mathbb{R})$ under dimensional reduction. In going from four to three dimensions the four-dimensional conformal group $S U(2,2)$ goes over to its $\operatorname{subgroup} S p(4, \mathbb{R})$. The maximal compact subgroup $S U(2)_{L} \times S U(2)_{R} \times U(1)$ of $S U(2,2)$ gets then restricted to the diagonal subgroup $S U(2) \times U(1)$. Thus in going down to three dimensions we must identify the spinors of $S U(2)_{L}$ and $S U(2)_{R}$,

$$
a_{i} \cong b_{i}:=c_{i}, \quad a^{i} \cong b^{i}:=c^{i} .
$$

Hence

$$
a_{i} b_{j} \rightarrow c_{i} c_{j} \quad, \quad a^{i} b^{j} \rightarrow c^{i} c^{j} \quad, \quad a^{i} a_{j} \cong b^{i} b_{j} \rightarrow c^{i} c_{j} .
$$

We get a singletonic realization of $S p(4, \mathbb{R})$ in three dimensions. Then the coherent states describing the massless conformal fields in four dimensions reduce to scalars and spin- $\frac{1}{2}$ fields in three dimensions as tabulated in Table 2.

We see that we get 128 massless scalar fields transforming in the $\mathbf{1 2 8}_{s}$ of $S O(16)$ and 128 massless spin $\frac{1}{2}$ fields transforming in $\mathbf{1 2 8}_{c}$ of $S O(16)$. They can be identified with the fields of maximal $\mathcal{N}=16$ supergravity in three dimensions. 


\begin{tabular}{|c|c|}
\hline$d=4$ Fields & $d=3$ Fields \\
\hline$\phi^{[A B C D]}(x)$ & $\phi^{[A B C D]}(x)$ \\
\hline$\lambda_{\alpha}^{[A B C]}(x)$ & $\psi_{\alpha}^{[A B C]}(x)$ \\
\hline$\lambda_{\dot{\alpha}[A B C]}(x)$ & $\psi_{\alpha[A B C]}(x)$ \\
\hline$h_{(\alpha \beta)}^{[A B]}(x)$ & $\partial_{\alpha \beta} \phi^{[A B]}(x)$ \\
\hline$h_{(\dot{\alpha} \dot{\beta})[A B]}(x)$ & $\partial_{\alpha \beta} \phi_{[A B]}(x)$ \\
\hline$\psi_{(\alpha \beta \gamma)}^{A}(x)$ & $\partial_{(\alpha \beta} \psi_{\gamma)}^{A}(x)$ \\
\hline$\psi_{(\dot{\alpha} \dot{\beta} \dot{\gamma}) A}(x)$ & $\partial_{(\alpha \beta} \psi_{\gamma) A}(x)$ \\
\hline$R_{(\alpha \beta \gamma \delta)}(x)$ & $\partial_{(\alpha \beta} \partial_{\gamma \delta)} \phi^{+}(x)$ \\
\hline$R_{(\dot{\alpha} \dot{\beta} \dot{\gamma} \dot{\delta})}(x)$ & $\partial_{(\alpha \beta} \partial_{\gamma \delta)} \phi^{-}(x)$ \\
\hline
\end{tabular}

Table 2: Three-dimensional decomposition of the CPT-self-conjugate doubleton supermultiplet of four-dimensional superconformal group $S U(2,2 \mid 8)$ under dimensional reduction. $A, B, C, .$. are the $S U(8)$ indices. Four-dimensional field-strengths yield scalars with derivatives in three dimensions.

\subsection{Three-dimensional constrained on-shell superfields}

We will now show that the fields of three-dimensional $\mathcal{N}=16$ supergravity can be conveniently organized in an on-shell superfield. We can introduce a suitable three-dimensional superspace with coordinates [54],

$$
\left(x^{\alpha \beta}, \theta^{\alpha \hat{a}}\right) \quad \text { with } \quad \alpha, \beta=1,2 \quad \hat{a}=1,2, \ldots, 16 .
$$

The bosonic coordinates $x^{\alpha \beta}$ are real symmetric matrices in the spinor indices. The fermionic coordinates $\theta^{\alpha \hat{a}}$ are Majorana Lorentz spinors and transform in the 16-dimensional vector representation of $S O(16)$. All the $R$-symmetry indices will be hatted throughout this section. One can introduce the superspace covariant derivatives,

$$
D_{\alpha \hat{a}}=\partial_{\alpha \hat{a}}+i \theta_{\hat{a}}^{\beta} \partial_{\alpha \beta}
$$

With this definition, the covariant derivatives obey the relation

$$
\left\{D_{\alpha \hat{a}}, D_{\beta \hat{b}}\right\}=2 i \delta_{\hat{a} \hat{b}} \partial_{\alpha \beta}
$$

We can then introduce the superfield

$$
\Phi_{\hat{\alpha}}\left(x^{\alpha \beta}, \theta^{\alpha \hat{a}}\right)
$$

which transforms as a Lorentz scalar and as a (real) $\mathbf{1 2 8}_{s}$ spinor of $S O(16)$. If $\hat{\alpha}=$ $1,2, \ldots 256$ is an $S O(16)$ spinor index, the superfield needs to obey the $S O(16)$ chirality 
condition

$$
\Gamma_{17} \Phi=+\Phi
$$

In order to have the right number of degrees of freedom in the superfield, we also need to impose the differential constraint,

$$
D_{\alpha}^{\hat{a}} \Phi_{\hat{\alpha}}=\frac{1}{16}\left(\Gamma^{\hat{a}} \Gamma^{\hat{b}}\right)_{\hat{\alpha}}^{\hat{\beta}} D_{\alpha}^{\hat{b}} \Phi_{\hat{\beta}}
$$

where $\Gamma^{\hat{a}}$ are $S O(16), 256 \times 256$ gamma matrices. We can define an additional superfield

$$
\Psi_{\alpha \hat{\alpha}}=\frac{1}{16}\left(\Gamma^{\hat{a}}\right)_{\hat{\alpha}}^{\hat{\beta}} D_{\alpha}^{\hat{a}} \Phi_{\hat{\beta}}
$$

With this definitions, all the 128 bosonic fields belong to the $\theta^{0}$ term of the expansion of the superfield $\Phi_{\hat{\alpha}}$ and all the 128 fermionic fields belong to the $\theta^{0}$ term of the superfield $\Psi_{\alpha \hat{\alpha}}$.

Due to the presence of the gamma matrix in the definition above, if the superfield $\Phi_{\hat{\alpha}}$ transforms in the $\mathbf{1 2 8}_{s}$ representation of $S O(16)$, the superfield $\Psi_{\alpha \hat{\alpha}}$ will transform in the $\mathbf{1 2 8}_{c}$ representation and vice-versa. We can then rewrite the differential constraint (3.32) as

$$
D_{\alpha}^{\hat{a}} \Phi_{\hat{\alpha}}=\left(\Gamma^{\hat{a}}\right)_{\hat{\alpha}}^{\hat{\beta}} \Psi_{\alpha \hat{\beta}} .
$$

Using the relations (3.29) and (3.32), we obtain a differential constraint for the superfield $\Psi_{\alpha \hat{\alpha}}$,

$$
D_{\alpha}^{\hat{a}} \Psi_{\beta \hat{\alpha}}=i\left(\Gamma^{\hat{a}}\right)_{\hat{\alpha}}^{\hat{\beta}} \partial_{\alpha \beta} \Phi_{\hat{\beta}}
$$

in agreement with the constraints imposed in [57]. Note that analogous constraints are also imposed in the $\mathcal{N}=8$ case [58]. The expansions of both superfields contain terms up to $\theta^{32}$. However, due to the relations (3.34) and (3.35), all terms with more than one fermionic coordinate do not contain any new degrees of freedom.

Moreover, taking the $\theta^{0}$ components of (3.34) and (3.35) we obtain the supersymmetry transformations of the component fields of the $\mathcal{N}=16$ multiplet,

$$
\begin{aligned}
\delta_{\eta} \phi_{\hat{\alpha}} & =\eta^{\hat{a} \hat{\alpha}}\left(\Gamma^{\hat{a}}\right)_{\hat{\alpha}}^{\hat{\beta}} \psi_{\alpha \hat{\beta}} \\
\delta_{\eta} \psi_{\beta \hat{\alpha}} & =i \eta^{\hat{a} \hat{\alpha}}\left(\Gamma^{\hat{a}}\right)_{\hat{\alpha}}^{\hat{\beta}} \partial_{\alpha \beta} \phi_{\hat{\beta}}
\end{aligned}
$$

where $\phi_{\hat{\alpha}}$ and $\psi_{\alpha \hat{\alpha}}$ are the bosonic and fermionic fields of the supermultiplet. 


\section{Unitary representations of $O S p\left(8^{*} \mid 8\right)$ and maximal supergravity in six dimensions}

\subsection{Coherent states of the positive energy unitary representations of $S O^{*}(8)$ and conformal fields in six dimensions}

Our main goal in this section is to construct the six-dimensional counterpart of the CPTself-conjugate doubleton supermultiplet of $S U(2,2 \mid 8)$ and discuss how it may be related to maximal supergravity in six dimensions. To this end we shall first review, following $[4,55,56]$, the oscillator construction of the positive energy unitary representations of the conformal group in six dimensions and its supersymmetric extensions.

The generators of the conformal group $S O(6,2)$ in $d=6$ satisfy the commutation relations

$$
\left[M_{a b}, M_{c d}\right]=i\left(\eta_{b c} M_{a d}-\eta_{a c} M_{b d}-\eta_{b d} M_{a c}+\eta_{a d} M_{b c}\right),
$$

where $a, b, c, d=0,1, \ldots, 7$ and $\eta_{a b}=\operatorname{diag}(+,-,-,-,-,-,-,+) . \quad M_{\mu \nu}$, with $\mu, \nu=$ $0,1, \ldots, 5$, are the generators of the Lorentz subgroup $S O(5,1)$. The dilatation generator $D$, the generators of translations $P_{\mu}$ and the special conformal generators $K_{\mu}$ are related to the generators above by

$$
M_{\mu 6}=\frac{1}{2}\left(P_{\mu}-K_{\mu}\right), \quad M_{\mu 7}=\frac{1}{2}\left(P_{\mu}+K_{\mu}\right), \quad M_{67}=-D,
$$

The covering group of the conformal group $S O(6,2)$ is $\operatorname{Spin}(6,2)$ which is isomorphic to $S O^{*}(8)$ and the covering group of the Lorentz group $S O(5,1)$ is $S U^{*}(4)$. The rotation subgroup $S O(5)$ (or its covering group $U S p(4)$ ) is generated by $M_{\mu \nu}$, with $\mu, \nu=1,2, \ldots, 5$.

The generators $M_{m n}(m, n=1,2, \ldots, 6)$ generate the compact subgroup $S U(4)$ and the $U(1)_{E}$ generator $E \equiv M_{07}$ is the conformal Hamiltonian.

The Lie algebra of the conformal group $S O(6,2)$ has a three-graded decomposition with respect to its maximal compact subalgebra $\mathcal{L}^{0}=S U(4) \times U(1)_{E}$,

$$
S O(6,2)=\mathcal{L}^{-} \oplus \mathcal{L}^{0} \oplus \mathcal{L}^{+},
$$

where the three-grading is determined by the conformal Hamiltonian $E=\frac{1}{2}\left(P_{0}+K_{0}\right)$, To construct positive energy unitary representations of $S O^{*}(8)$, one realizes the generators as bilinears of an arbitrary number $P$ of pairs of bosonic annihilation $\left(\mathbf{a}_{i}, \mathbf{b}_{j}\right)$ and creation $\left(\mathbf{a}^{i}, \mathbf{b}^{j}\right)$ operators (with $\left.i, j=1,2,3,4\right)$, transforming in the fundamental representation of $S U(4)$ and its conjugate, respectively [4, 55, 59,60,61],

$$
\begin{aligned}
A_{i j} & :=\mathbf{a}_{i} \cdot \mathbf{b}_{j}-\mathbf{a}_{j} \cdot \mathbf{b}_{i}, \\
A^{i j} & :=\mathbf{a}^{i} \cdot \mathbf{b}^{j}-\mathbf{a}^{j} \cdot \mathbf{b}^{i}, \\
M_{j}^{i} & :=\mathbf{a}^{i} \cdot \mathbf{a}_{j}+\mathbf{b}_{j} \cdot \mathbf{b}^{i} .
\end{aligned}
$$


The dot product denotes contraction with respect to the color or generation index, i.e. $\mathbf{a}_{i} \cdot \mathbf{b}_{j}:=\sum_{R=1}^{P} a_{i}(R) b_{j}(R)$. The bosonic annihilation and creation operators $a^{i}(R)=$ $a_{i}(R)^{\dagger}$ and $b^{j}(R)=b_{j}(R)^{\dagger}$ satisfy the usual canonical commutation relations

$$
\left[a_{i}(R), a^{j}(S)\right]=\delta_{i}^{j} \delta_{R S} \quad, \quad\left[b_{i}(R), b^{j}(S)\right]=\delta_{i}^{j} \delta_{R S}
$$

where $i, j=1,2,3,4$ and $R, S=1,2, \ldots, P$.

$M^{i}{ }_{j}$ generate the maximal compact subgroup $U(4)$. The conformal Hamiltonian is given by the trace $M^{i}{ }_{i}$

$$
Q_{B}:=\frac{1}{2} M_{i}^{i}=\frac{1}{2}\left(N_{B}+4 P\right)
$$

where $N_{B} \equiv \mathbf{a}^{i} \cdot \mathbf{a}_{i}+\mathbf{b}^{i} \cdot \mathbf{b}_{i}$, which is the bosonic number operator. We shall denote the eigenvalues of $Q_{B}$ as $E$. The hermitian linear combinations of $A_{i j}$ and $A^{i j}$ are the non-compact generators of $S O(6,2)[4,55,59]$. Each lowest weight (positive energy) UIR is uniquely determined by a set of states transforming in the lowest energy irreducible representation $|\Omega\rangle$ of $S U(4) \times U(1)_{E}$ that are annihilated by all the elements of $\mathcal{L}^{-}[4,55] .{ }^{10}$ The possible lowest weight vectors for $P=1$, which are called doubleton representations [55], in this compact basis, are of the form

$$
\begin{aligned}
& |0\rangle \text {, } \\
& a^{i_{1}}|0\rangle=|\square\rangle, \\
& a^{\left(i_{1}\right.} a^{\left.i_{2}\right)}|0\rangle=|\square \square\rangle, \\
& \vdots
\end{aligned}
$$

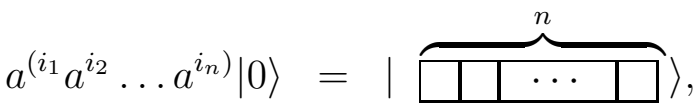

together with those obtained by interchanging $a$-type oscillators with $b$-type oscillators and together with the state

$$
a^{(i} b^{j)}|0\rangle=|\square \square\rangle
$$

These lowest energy irreducible representations $|\Omega\rangle$ - or doubleton UIRs - of $S O^{*}(8)$ all transform in the symmetric tensor representations of $S U(4)$.

Conformal fields in six dimensions transform covariantly under the Lorentz group $S U^{*}(4)=\operatorname{Spin}(5,1)$ and have definite conformal dimension. Similarly to the fourdimensional conformal group discussed in Section 2, the conformal group $S O(6,2)$ also has a non-compact three-graded structure with respect to its subgroup $S U^{*}(4) \times \mathcal{D}$, where

\footnotetext{
${ }^{10}$ Equivalently, the lowest weight vector of the lowest energy irreducible representation of $S U(4)$ determines the UIR. Hence, by an abuse of terminology, we shall use interchangeably the terms "lowest weight vector" and "lowest energy irreducible representation".
} 
$\mathcal{D}$ is the dilation generator $[55,62]$. Under the action of $S O(6,2)$ on the (conformal compactification of) six-dimensional Minkowski spacetime, the stability group $\mathcal{H}$ of the origin $x^{\mu}=0$ is the semi-direct product

$$
\left(S U^{*}(4) \times \mathcal{D}\right)(S) \mathcal{K}_{6}
$$

where $\mathcal{K}_{6}$ represents the Abelian subgroup generated by the special conformal generators $K_{\mu}$. Conformal fields in $d=6$ live on the coset space $G / \mathcal{H}$ and are labeled by their transformation properties under the Lorentz group $S U^{*}(4)$, their conformal (scale) dimension $l$ and certain matrices $\kappa_{\mu}$ that describe their behavior under special conformal transformations $K_{\mu}$ [55], in complete parallel the four-dimensional situation reviewed in Section $2[42,43]$.

Representations of the six-dimensional conformal algebra may be conveniently constructed in terms of the twistorial spinor

$$
\Psi(R):=\left(\begin{array}{c}
a_{i}(R) \\
b^{j}(R)
\end{array}\right)
$$

and its Dirac conjugate

$$
\bar{\Psi}(R) \equiv \Psi^{\dagger}(R) \Gamma_{0}=\left(\begin{array}{ll}
a^{i}(R) & -b_{j}(R)
\end{array}\right) .
$$

The generators of $S O(6,2)$ can be written as bilinears of these twistorial oscillators

$$
\overline{\mathbf{\Psi}} \Sigma_{a b} \Psi:=\sum_{R=1}^{P} \bar{\Psi}(R) \Sigma_{a b} \Psi(R),
$$

and satisfy the commutation relations

$$
\left[\overline{\boldsymbol{\Psi}} \Sigma_{a b} \boldsymbol{\Psi}, \overline{\boldsymbol{\Psi}} \Sigma_{c d} \boldsymbol{\Psi}\right]=\overline{\mathbf{\Psi}}\left[\Sigma_{a b}, \Sigma_{c d}\right] \boldsymbol{\Psi},
$$

as a consequence of the the canonical commutation relations (4.5) of the oscillators $a_{i}$ and $b_{j}$. The matrices $\Sigma_{a b}$ are defined in terms of the six-dimensional Dirac matrices $\Gamma_{\mu}$, with $\left\{\Gamma_{\mu}, \Gamma_{\nu}\right\}=2 \eta_{\mu \nu}$, as

$$
\Sigma_{\mu \nu}:=\frac{i}{4}\left[\Gamma_{\mu}, \Gamma_{\nu}\right], \quad \Sigma_{\mu 6}:=-\frac{1}{2} \Gamma_{\mu} \Gamma_{7}, \quad \Sigma_{\mu 7}:=\frac{1}{2} \Gamma_{\mu}, \quad \Sigma_{67}:=\frac{i}{2} \Gamma_{7},
$$

where $\Gamma_{7}=-\Gamma_{0} \Gamma_{1} \Gamma_{2} \Gamma_{3} \Gamma_{4} \Gamma_{5}$. The $\Sigma$ matrices generate the eight-dimensional "lefthanded" spinor representation of the conformal algebra $S O(6,2)^{11}$. The Lorentz covariant generators of the conformal algebra are

$$
\begin{aligned}
M_{\mu \nu} & =\frac{i}{4} \bar{\Psi}\left[\Gamma_{\mu}, \Gamma_{\nu}\right] \Psi, & D & =-\frac{i}{2} \bar{\Psi} \Gamma_{7} \Psi, \\
P_{\mu} & =\frac{1}{2} \bar{\Psi} \Gamma_{\mu}\left(I-\Gamma_{7}\right) \Psi, & K_{\mu} & =\frac{1}{2} \bar{\Psi} \Gamma_{\mu}\left(I+\Gamma_{7}\right) \Psi
\end{aligned}
$$

\footnotetext{
${ }^{11}$ Our conventions for $\Gamma$ matrices follow $[56]$ and are outlined in Appendix B.
} 
The positive energy UIRs of $S O^{*}(8)$ can be identified with conformal fields in six dimensions, transforming covariantly under the six-dimensional Lorentz group and with definite conformal dimension and trivial special conformal parameters $\kappa_{\mu}$. To establish this correspondence consider the operator

$$
T:=e^{\frac{\pi}{2} \bar{\Psi} \Sigma_{06} \Psi}
$$

which satisfies the following important relations :

$$
\begin{aligned}
M_{m n} T & =T M_{m n} \quad \text { for } m, n=1,2, \ldots, 5 \\
i M_{m 0} T & =T M_{m 6}, \\
i D T & =T E, \\
K_{\mu} T & =T \mathcal{L}^{-},
\end{aligned}
$$

where $\mathcal{L}^{-}$stands for certain linear combinations of the operators $A_{i j}$. Therefore, similarly to the intertwiner of the four-dimensional conformal algebra given in equation (2.6), $T$ in equation (4.16) intertwines between the generators $\left(M_{\mu \nu}, D\right)$ of Lorentz group and dilatations and the generators $\left(M_{m n}, E\right)$ of the maximal compact subgroup $S U(4) \times U(1)$. Thus, the transformation properties of the states $|\Omega\rangle$ that make up the minimal energy irreducible representation of $S U(4)$ coincide with the transformation properties of $T|\Omega\rangle$ under the Lorentz group $S U^{*}(4)$.

Therefore every unitary lowest weight representation (ULWR) of $S O^{*}(8)$ can be identified with a unitary representation of $S O(6,2)$ induced by a finite dimensional irreducible representation of $S U^{*}(4)$ with a definite conformal dimension $l$ and trivially realized $K_{\mu} \cdot{ }^{12}$ The states constructed above do not carry any position dependence, i.e. they are located at $x^{\mu}=0$. As discussed in Section 2 in a four-dimensional context, a state at any other space-time point is generated by the action of the translation operator:

$$
e^{i x^{\mu} P_{\mu}} T|\Omega\rangle=\left|\Phi_{\left(d_{1}, d_{2}, d_{3}\right)}(x)\right\rangle
$$

where $\left(d_{1}, d_{2}, d_{3}\right)$ are the Dynkin labels. Thus, every irreducible ULWR of $S O(6,2)$ corresponds to a conformal field that transforms covariantly under $S U^{*}(4)$ with a definite conformal dimension $l=-E$. These coherent states correspond to six-dimensional conformal fields.

The doubleton representations of $S O^{*}(8)$ are constructed using a single pair $(P=1)$ of bosonic oscillators. The Poincaré mass operator

$$
M^{2}=P_{\mu} P^{\mu}
$$

\footnotetext{
${ }^{12}$ This follows from the fact that $\mathcal{L}^{-}|\Omega\rangle=0$ implies that $K_{\mu} T|\Omega\rangle=0[55]$.
} 
in six-dimensional Minkowski spacetime vanishes identically for the doubleton representations and hence the corresponding conformal fields are massless. Below we shall restrict ourselves to the massless (doubleton) representations of the conformal group ${ }^{13}$.

The $S U^{*}(4)$ covariant spinorial oscillators are obtained from the $S U(4)$ covariant oscillators by the action of intertwining operator $T$. We will use hatted Greek letters for the $S U^{*}(4)$ indices $-\hat{\alpha}, \hat{\beta}=1,2,3,4$. With this notation, the $S U^{*}(4)$ covariant spinorial oscillators are:

$$
\begin{aligned}
& \lambda^{\hat{\alpha} 1}=\frac{1}{\sqrt{2}} T\left(\begin{array}{l}
a^{1} \\
a^{2} \\
a^{3} \\
a^{4}
\end{array}\right) T^{-1}=\frac{1}{\sqrt{2}}\left(\begin{array}{l}
a^{1}-b_{3} \\
a^{2}+b_{4} \\
a^{3}+b_{1} \\
a^{4}-b_{2}
\end{array}\right), \\
& \lambda^{\hat{\alpha} 2}=\frac{1}{\sqrt{2}} T\left(\begin{array}{l}
b^{1} \\
b^{2} \\
b^{3} \\
b^{4}
\end{array}\right) T^{-1}=\frac{1}{\sqrt{2}}\left(\begin{array}{l}
b^{1}+a_{3} \\
b^{2}-a_{4} \\
b^{3}-a_{1} \\
b^{4}+a_{2}
\end{array}\right), \\
& \eta_{\hat{\alpha} 1}=\frac{1}{\sqrt{2}} T\left(\begin{array}{l}
a_{1} \\
a_{1}+b^{3} \\
a_{3} \\
a_{2}-b^{4} \\
a_{3}-b^{1} \\
a_{4}+b^{2}
\end{array}\right) T^{-1}=\frac{1}{\sqrt{2}}\left(\begin{array}{l}
b_{1}-a^{3} \\
b_{2}+a^{4} \\
b_{3}+a^{1} \\
b_{4}-a^{2}
\end{array}\right) .
\end{aligned}
$$

As a consequence of equation (4.5), they satisfy canonical commutation relations

$$
\left[\eta_{\hat{\alpha} i}, \lambda^{\hat{\beta} j}\right]=\frac{1}{2} \delta_{\hat{\alpha}}^{\hat{\beta}} \delta_{i}^{j}
$$

where $i, j=1,2$ and, as mentioned above, $\hat{\alpha}, \hat{\beta}=1,2,3,4$. Then we find that

$$
\left(\Sigma^{\mu}\right)^{\hat{\alpha} \hat{\beta}} P_{\mu}=-2 \lambda^{\hat{\alpha} i} \lambda^{\hat{\beta} j} \epsilon_{i j} \quad, \quad\left(\Sigma^{\mu}\right)_{\hat{\alpha} \hat{\beta}} K_{\mu}=-2 \eta_{\hat{\alpha} i} \eta_{\hat{\beta} j} \epsilon^{i j}
$$

\footnotetext{
${ }^{13}$ Recently it was shown that massless doubleton representations correspond to the minimal unitary representation of $S O^{*}(8)$ and its deformations are labelled by $S U(2)$ spin, which is the six-dimensional analog of helicity in four dimensions [9].
} 
where $\Sigma$-matrices in $d=6$ are the analogs of Pauli matrices $\sigma_{\mu}$ in $d=4 .{ }^{14}$

$$
\bar{\Sigma}_{\mu}=\left(\Sigma_{0},-\Sigma_{1},-\Sigma_{2},-\Sigma_{3},-\Sigma_{4},-\Sigma_{5}\right)
$$

The first equation above is similar to the one used in the six-dimensional spinor helicity formalism [63, 64]. The doubleton irreducible representations of $S O(6,2)$ and the corresponding conformal fields are listed in Table 3. Thus the massless conformal fields in six dimensions can be labelled as symmetric tensors in the spinor indices as $\Phi_{A B C \ldots E}(x)$ corresponding to a Dynkin label $(n, 0,0)$ of $S U^{*}(4)$. This shows that the massless graviton, which transforms in the 20-dimensional representation of $S U^{*}(4)$ with Dynkin label $(0,2,0)$, cannot be a massless conformal field. Similarly, a massless vector field in six dimensions cannot be conformal since it transforms in the $(0,1,0)$ representation of $S U^{*}(4)$.

\begin{tabular}{|c|c|c|}
\hline $\begin{array}{l}\text { States of lowest energy } \\
\text { irreps of } S U(4)\end{array}$ & $\begin{array}{l}\qquad U^{*}(4) \text { Dynkin labels } \\
\text { coherent states }\left|\Phi_{\left(d_{1}, d_{2}, d_{3}\right)}(x)\right\rangle\end{array}$ & $\begin{array}{c}\text { conformal dimension } \\
l \\
\end{array}$ \\
\hline$|0\rangle$ & $\left|\Phi_{(0,0,0)}(x)\right\rangle$ & -2 \\
\hline$a^{\hat{i}_{1}}|0\rangle$ & $\left|\Phi_{(1,0,0)}(x)\right\rangle$ & $-\frac{5}{2}$ \\
\hline$a^{\left(\hat{i}_{1}\right.} a^{\left.\hat{i}_{2}\right)}|0\rangle$ & $\left|\Phi_{(2,0,0)}(x)\right\rangle$ & -3 \\
\hline$\vdots$ & $\vdots$ & $\vdots$ \\
\hline$a^{\left(\hat{i}_{1}\right.} \ldots a^{\left.\hat{i}_{n}\right)}|0\rangle$ & $\left|\Phi_{(n, 0,0)}(x)\right\rangle$ & $-\frac{1}{2}(n+4)$ \\
\hline$a^{\left(\hat{i}_{1}\right.} b^{\left.\hat{j}_{2}\right)}|0\rangle$ & $\left|\Phi_{(2,0,0)}(x)\right\rangle$ & -3 \\
\hline
\end{tabular}

Table 3: States transforming in the lowest energy irreducible representation of $S U(4)$ in the compact basis with their $S U^{*}(4)$ Dynkin labels and conformal (scale) dimensions.

\subsection{Six-dimensional conformal superalgebras $O S p\left(8^{*} \mid 2 N\right)$}

Simple conformal superalgebras in six dimensions belong to the family $O S p\left(8^{*} \mid 2 N\right)$ with the even subgroup $S O^{*}(8) \times U S p(2 N)$. They correspond to chiral $(N, 0)$ supersymmetry since the odd generators belong to a spinorial representation of definite chirality ${ }^{15}$.

The superalgebra $O S p\left(8^{*} \mid 4\right)$ appearing at $N=2$ may be interpreted both as the $\mathcal{N}=4$ extended $A d S$ superalgebra in $d=7$ or as the $(2,0)$ extended conformal superalgebra with 32 supercharges in six dimensions. The interacting quantum theory of the $(2,0)$ doubleton supermultiplet of $O S p\left(8^{*} \mid 4\right)$ is believed to be dual to $M$-theory over $A d S_{7} \times S^{4}$.

\footnotetext{
${ }^{14}$ The explicit form of the $\Sigma_{\mu}$ matrices is given in Appendix B.

${ }^{15}$ Actually due to triality properties of $S O(8)$, there exist three different forms of these superalgebras which are in triality.
} 
The supersymmetry generators $Q_{\Gamma A}$ of $O S p\left(8^{*} \mid 2 N\right)$ satisfy the anticommutation relations [65]

$$
\left\{Q_{\Gamma A}, Q_{\Delta B}\right\}=-\frac{1}{2}\left(\Omega_{A B} M_{\Gamma \Delta}+C_{\Gamma \Delta} U_{A B}\right),
$$

where $A, B, . .=1, . ., 2 N$ and $\Gamma, \Delta=1, \ldots 8 . U_{A B}=U_{B A}$ are the $U S p(2 N)$ generators and $\Omega_{A B}=-\Omega_{B A}$ is the symplectic invariant tensor. The tensor $C_{\Gamma \Delta}$ is the charge conjugation matrix in $(6,2)$ dimensions and is symmetric [65].

The generators of $U S p(2 N)$ satisfy

$$
\left[U_{A B}, U_{C D}\right]=\Omega_{A(C} U_{D) B}+\Omega_{B(C} U_{D) A} .
$$

The commutation relations of $S O(6,2)$ and $U S p(2 N)$ with the supersymmetry generators,

$$
\begin{gathered}
{\left[M_{a b}, Q_{\Gamma A}\right]=\left(\Sigma_{a b}\right)_{\Gamma}{ }^{\Delta} Q_{\Delta A},} \\
{\left[U_{A B}, Q_{C \Gamma}\right]=-\Omega_{C(A} Q_{B) \Delta},}
\end{gathered}
$$

identify the supercharges $Q_{\Delta A}$ as the bi-fundamental representation of the bosonic subgroup.

The superalgebra $O S p\left(8^{*} \mid 2 N\right)$ has a three-graded decomposition with respect to its compact subsuperalgebra $\mathcal{L}^{0}=U(4 \mid N)$,

$$
O S p\left(8^{*} \mid 2 N\right)=\mathcal{L}^{+} \oplus \mathcal{L}^{0} \oplus \mathcal{L}^{-},
$$

This three-grading restricts to the three-grading of $S O^{*}(8)$ with respect to $U(4)$ subgroup and to the three-grading of $U S p(2 N)$ with respect to its $U(N)$ subgroup.

The Lie superalgebra $O S p\left(8^{*} \mid 2 N\right)$ is then realized as bilinears of super-oscillators transforming in the fundamental representation of $U(4 \mid N)$ and its conjugate, respectively. The superoscillators are defined as

$$
\begin{array}{ll}
\xi_{\hat{A}}(R)=\left(\begin{array}{c}
a_{i}(R) \\
\alpha_{x}(R)
\end{array}\right), & \xi^{\hat{A}}(R)=\left(\begin{array}{c}
a^{i}(R) \\
\alpha^{x}(R)
\end{array}\right) \\
\eta_{\hat{A}}(R)=\left(\begin{array}{c}
b_{i}(R) \\
\beta_{x}
\end{array}\right), & \eta^{\hat{A}}(R)=\left(\begin{array}{c}
b^{i}(R) \\
\beta^{x}(R)
\end{array}\right)
\end{array}
$$

where $i=1, \ldots, 4, x=1, \ldots, N$ and $R=1, \ldots, P$. The oscillators satisfy the usual graded commutation relations,

$$
\left[\xi_{\hat{A}}(R), \xi^{\hat{B}}(S)\right\}=\delta_{\hat{A}}^{\hat{B}} \delta_{R S}, \quad\left[\eta_{\hat{A}}(R), \eta^{\hat{B}}(S)\right\}=\delta_{\hat{A}}^{\hat{B}} \delta_{R S} .
$$

Then, the grade +1 , grade 0 and grade -1 generators respectively have the following expressions

$$
\begin{aligned}
A^{\hat{A} \hat{B}} & :=\boldsymbol{\eta}^{\hat{B}} \cdot \boldsymbol{\xi}^{\hat{A}}+\boldsymbol{\xi}^{\hat{B}} \cdot \boldsymbol{\eta}^{\hat{A}} \\
M_{\hat{B}}^{\hat{A}} & :=\boldsymbol{\xi}^{\hat{A}} \cdot \boldsymbol{\xi}_{\hat{B}}+(-1)^{(\operatorname{deg} \hat{A})(\operatorname{deg} \hat{B})} \boldsymbol{\eta}_{\hat{B}} \cdot \boldsymbol{\eta}^{\hat{A}} \\
A_{\hat{A} \hat{B}} & :=\boldsymbol{\xi}_{\hat{A}} \cdot \boldsymbol{\eta}_{\hat{B}}+\boldsymbol{\eta}_{\hat{A}} \cdot \boldsymbol{\xi}_{\hat{B}} .
\end{aligned}
$$


The resulting unitary supermultiplets of $O S p\left(8^{*} \mid 2 N\right)$ in the super-Fock space decompose into a finite set of positive energy irreducible representations of $S O^{*}(8)$ transforming in irreducible representations of $U S p(2 N)$. For a single pair of super-oscillators $P=1$ (doubletons) the resulting representations correspond to superconformal multiplets of massless fields in six dimensions.

\begin{tabular}{|c|c|c|}
\hline Conformal Field & $S U^{*}(4)_{D}$ & $U S p(4)$ \\
\hline$\phi^{[A B] \mid}(x)$ & $(0,0,0)$ & 5 \\
\hline$\lambda_{\hat{\alpha}}^{A}(x)$ & $(1,0,0)$ & 4 \\
\hline$h_{(\hat{\alpha} \hat{\beta})}(x)$ & $(2,0,0)$ & 1 \\
\hline
\end{tabular}

Table 4: Doubleton supermultiplet of $O S p\left(8^{*} \mid 4\right)$ corresponding to the lowest weight vector $|\Omega\rangle=$ $|0\rangle$, with $S U^{*}(4)$ Dynkin labels and dimension of the $U S p(4)$ representations. The conformal fields in the first column are labelled by $S U^{*}(4)$ spinor indices $\hat{\alpha}, \hat{\beta}, \ldots$ and $U S p(4)$ indices $A, B, \ldots$ The square bracket $[\cdots] \mid$ denote an antisymmetric, symplectic-traceless tensor.

The doubleton supermultiplet of $O S p\left(8^{*} \mid 4\right)$, which is defined by the lowest weight vector $|\Omega\rangle=|0\rangle$, is the massless $(2,0)$ conformal supermultiplet and is the analog of the $\mathcal{N}=4$ super Yang-Mills multiplet in $d=6$ [4]. The content of the $(2,0)$ supermultiplet is given in Table 4.

In Table 5 we give the field content of the doubleton supermultiplet of $O S p\left(8^{*} \mid 8\right)$ with $R$-symmetry group $U S p(8)$, namely the $(4,0)$ conformal supermultiplet. The oscillator construction of the positive energy unitary supermultiplets of $O S p\left(2 M^{*} \mid 2 N\right)$ with the even subgroup $S O^{*}(2 M) \times U S p(2 N)$ was given in [66]. For both the $(2,0)$ and the $(4,0)$ supermultiplets, the oscillator construction gives directly the gauge-invariant field strengths. The corresponding gauge potentials are discussed in Section 4.6.

\subsection{Constrained on-shell superfields for the six-dimensional $(4,0)$ supermul- tiplet}

The fields of the $(4,0)$ supermultiplet in six dimensions can be organized in an on-shell superfield obeying two simple algebraic and differential constraints. Following the conventions in [67] (see also [50, 68]) the coordinates of the six-dimensional extended superspace are

$$
\left(x^{\hat{\alpha} \hat{\beta}}, \theta_{A}^{\hat{\alpha}}\right) \quad \hat{\alpha}, \hat{\beta}=1, \ldots, 4 ; \quad A=1, \ldots, 8 ;
$$

as mentioned previously, $S U^{*}(4)$ spinor indices are denoted by hatted Greek letters $\hat{\alpha}, \hat{\beta} \ldots$; we will denote $U S p(8) R$-symmetry indices by capital Latin letters $A, B, C \ldots$ 


\begin{tabular}{|c|c|c|}
\hline Conformal Field & $S U^{*}(4)_{D}$ & $U S p(8)$ \\
\hline$\phi^{[A B C D] \mid}(x)$ & $(0,0,0)$ & 42 \\
\hline$\lambda_{\hat{\alpha}}^{[A B C] \mid}(x)$ & $(1,0,0)$ & 48 \\
\hline$h_{(\hat{\alpha} \hat{\beta})}^{[A B] \mid}(x)$ & $(2,0,0)$ & 27 \\
\hline$\psi_{(\hat{\alpha} \hat{\beta} \hat{\gamma})}^{A}(x)$ & $(3,0,0)$ & 8 \\
\hline$R_{(\hat{\alpha} \hat{\beta} \hat{\gamma} \hat{\delta})}(x)$ & $(4,0,0)$ & 1 \\
\hline
\end{tabular}

Table 5: Doubleton supermultiplet of $\operatorname{OSp}\left(8^{*} \mid 8\right)$ corresponding to the lowest weight vector $|\Omega\rangle=|0\rangle$ with $S U^{*}(4)$ Dynkin labels and dimensions of the $U S p(8)$ representations. The conformal fields are labelled by $S U^{*}(4)$ spinor indices $\hat{\alpha}, \hat{\beta}, \ldots$ and $U S p(8)$ indices $A, B, \ldots$

The superspace covariant derivative is defined as

$$
D_{\hat{\alpha}}^{A}=\partial_{\hat{\alpha}}^{A}+i \Omega^{A B} \theta_{B}^{\hat{\beta}} \partial_{\hat{\alpha} \hat{\beta}},
$$

where $\partial_{\hat{\alpha}}^{A} \theta_{B}^{\hat{\beta}}=\delta_{B}^{A} \delta_{\hat{\alpha}}^{\hat{\beta}}$. In the above expression $\Omega_{A B}=-\Omega_{B A}$ is a symplectic matrix which obeys

$$
\Omega_{A B} \Omega^{B C}=\delta_{A}^{C} .
$$

The symplectic matrix $\Omega_{A B}$ can be used to raise or lower indices as follows,

$$
\theta^{A \hat{\alpha}}=\Omega^{A B} \theta_{B}^{\hat{\alpha}}, \quad D_{A \hat{\alpha}}=\Omega_{A B} D_{\hat{\alpha}}^{B} .
$$

With these definitions, the superspace derivatives obey the anticommutation relations

$$
\left\{D_{\hat{\alpha}}^{A}, D_{\hat{\beta}}^{B}\right\}=2 i \Omega^{A B} \partial_{\hat{\alpha} \hat{\beta}}, \quad\left\{D_{\hat{\alpha} A}, D_{\hat{\beta}}^{B}\right\}=2 i \delta_{A}^{B} \partial_{\hat{\alpha} \hat{\beta}} .
$$

To construct the superfield containing the fields of the $(4,0)$ multiplet we begin, following the analysis in [69] of the $(2,0)$ multiplet, with the superfield

$$
\Phi^{A B C D}\left(x^{\hat{\alpha} \hat{\beta}}, \theta_{A}^{\hat{\alpha}}\right)
$$

transforming in the 42 representation of $U S p(8)$, i.e. its $U S p(8)$ indices $A, B, C, D$ are antisymmetrized and obey a symplectic-traceless condition

$$
\Phi^{A B C D} \Omega_{C D}=0 .
$$

To have the right number of independent degrees of freedom we also need to impose a differential constraint of the form

$$
D_{\hat{\alpha}}^{A} \Phi^{B C D E}+\frac{1}{21} D_{\hat{\alpha} F}\left\{\Omega^{A[B} \Phi^{C D E] F}+\frac{3}{4} \Omega^{[B C} \Phi^{D E] A F}\right\}=0 .
$$


This differential constraint is analogous to the ones appearing in the superfield descriptions of the $(2,0)$ supermultiplet in six dimensions and of the $\mathcal{N}=8$ supermultiplet in four dimensions (Equation 2.38). The numerical coefficient $\frac{1}{21}$ in the second term is chosen such that (4.41) sets to zero all $U S p(8)$ representations appearing in the product $8 \times \mathbf{4 2}$ except for the 48-dimensional one. Thus, (4.41) eliminates all spin- $\frac{1}{2}$ fields except for those transforming in the 48 representation of $U S p(8)$. Furthermore, we can define the additional superfields

$$
\begin{aligned}
\Lambda_{\hat{\alpha}}^{A B C} & =\frac{2}{7} i D_{\hat{\alpha} D} \Phi^{D A B C} & H_{\hat{\alpha} \hat{\beta}}^{A B} & =-\frac{3}{16} D_{\hat{\alpha} C} \Lambda_{\hat{\beta}}^{C A B}, \\
\Psi_{\hat{\alpha} \hat{\beta} \hat{\gamma}}^{A} & =\frac{4}{27} i D_{\hat{\alpha} B} H_{\hat{\beta} \hat{\gamma}}^{B A}, & R_{\hat{\alpha} \hat{\beta} \hat{\gamma} \hat{\delta}} & =-\frac{1}{8} D_{\hat{\alpha} A} \Psi_{\hat{\beta} \hat{\gamma} \hat{\delta}}^{A} .
\end{aligned}
$$

They contain the same degrees of freedom as $\Phi^{A B C D}$ except that their lowest components are the component fields of the $(4,0)$ supermultiplet: $\lambda_{\hat{\alpha}}^{A B C}, h_{\hat{\alpha} \hat{\beta}}^{A B}, \psi_{\hat{\alpha} \hat{\beta} \hat{\gamma}}^{A}$ and $R_{\hat{\alpha} \hat{\beta} \hat{\gamma} \hat{\delta}}$, respectively ${ }^{16}$. It is not difficult to check that the superfields in equation (4.42) are symmetric in the spacetime indices.

The differential constraint (4.41) can be rewritten in terms of the superfields introduced in equation (4.42),

$$
D_{\hat{\alpha}}^{A} \Phi^{B C D E}=-\frac{i}{6}\left(\Omega^{A[B} \Lambda_{\hat{\alpha}}^{C D E]}+\frac{3}{4} \Omega^{[B C} \Lambda_{\hat{\alpha}}^{D E] A}\right) .
$$

This expression can be used to show that the superfields in (4.42) should themselves obey corresponding differential constraints,

$$
\begin{aligned}
D_{\hat{\alpha}}^{A} \Lambda_{\hat{\beta}}^{B C D} & =2 \partial_{\hat{\alpha} \hat{\beta}} \Phi^{A B C D}-\frac{1}{2} \Omega^{A[B} H_{\hat{\alpha} \hat{\beta}}^{C D]}+\frac{1}{6} \Omega^{[B C} H_{\hat{\alpha} \hat{\beta}}^{D] A} \\
D_{\hat{\alpha}}^{A} H_{\hat{\beta} \hat{\gamma}}^{B C} & =i \partial_{\hat{\alpha}(\hat{\beta}} \Lambda_{\hat{\gamma})}^{A B C}-i \Omega^{A[B} \Psi_{\hat{\alpha} \hat{\beta} \hat{\gamma}}^{C]}-\frac{i}{4} \Omega^{B C} \Psi_{\hat{\alpha} \hat{\beta} \hat{\gamma}}^{A} \\
D_{\hat{\alpha}}^{A} \Psi_{\hat{\beta} \hat{\gamma} \hat{\delta}}^{B} & =\frac{1}{3} \partial_{\hat{\alpha}(\hat{\beta}} H_{\hat{\gamma} \hat{\delta})}^{A B}-\Omega^{A B} R_{\hat{\alpha} \hat{\beta} \hat{\gamma} \hat{\delta}} \\
D_{\hat{\alpha}}^{A} R_{\hat{\beta} \hat{\gamma} \hat{\delta} \hat{\epsilon}} & =\frac{i}{12} \partial_{\hat{\alpha}(\hat{\beta}} \Psi_{\hat{\gamma} \hat{\delta} \hat{\epsilon})}^{A} .
\end{aligned}
$$

The normalization constants in (4.42) have been chosen to keep the numerical factors in the above expressions simple. Note that these differential constraints do not impose any further constraint on the lowest component fields $\lambda_{\hat{\alpha}}^{A B C}, h_{\hat{\alpha} \hat{\beta}}^{A B}, \psi_{\hat{\alpha} \hat{\beta} \hat{\gamma}}^{A}$ and $R_{\hat{\alpha} \hat{\beta} \hat{\gamma} \hat{\delta}}$, which all correspond to independent degrees of freedom transforming in the $48,27,8$ and singlet

\footnotetext{
${ }^{16}$ With potential abuse of notation, we will denote by $R$ both the six-dimensional field transforming in the $(4,0,0)$ representation of $S U^{*}(4)$ and the corresponding superfield. The field $R$ will be sometimes referred to as the generalized graviton field strength since it reduces to the four-dimensional Riemann tensor under dimensional reduction
} 
representations of $U S p(8)$ respectively. Moreover, if we expand the above differential constraints and take the $\theta^{0}$ components, we can read off the supersymmetry transformation rules of the fields of the $(4,0)$ supermultiplet,

$$
\begin{aligned}
\delta_{\eta} \phi_{\hat{\alpha}}^{A B C D} & =\frac{i}{6} \eta^{\hat{\alpha}[A} \lambda_{\hat{\alpha}}^{B C D]}-\frac{i}{8} \eta_{E}^{\hat{\alpha}} \Omega^{[A B} \lambda_{\hat{\alpha}}^{C D] E} \\
\delta_{\eta} \lambda_{\hat{\alpha}}^{A B C} & =2 \eta_{D}^{\hat{\beta}} \partial_{\hat{\alpha} \hat{\beta}} \phi^{A B C D}+\frac{1}{2} \eta^{\hat{\beta}[A} h_{\hat{\alpha} \hat{\beta}}^{B C]}+\frac{1}{6} \eta_{D}^{\hat{\beta}} \Omega^{[A B} h_{\hat{\alpha} \hat{\beta}}^{C] D} \\
\delta_{\eta} h_{\hat{\alpha} \hat{\beta}}^{A B} & =i \eta_{C}^{\hat{\gamma}} \partial_{\hat{\gamma}(\hat{\alpha}} \lambda_{\hat{\beta})}^{A B C}+i \eta^{\hat{\gamma}[A} \psi_{\hat{\alpha} \hat{\beta} \hat{\gamma}}^{B]}-\frac{i}{4} \eta_{C}^{\hat{\gamma}} \Omega^{A B} \psi_{\hat{\alpha} \hat{\beta} \hat{\gamma}}^{C}, \\
\delta_{\eta} \psi_{\hat{\alpha} \hat{\beta} \hat{\gamma}}^{A} & =-\frac{1}{3} \eta_{B}^{\hat{\delta}} \partial_{\hat{\delta}(\hat{\alpha}} h_{\hat{\beta} \hat{\gamma})}^{A B}+\eta^{\hat{\delta} A} R_{\hat{\alpha} \hat{\beta} \hat{\gamma} \hat{\delta}}, \\
\delta_{\eta} R_{\hat{\alpha} \hat{\beta} \hat{\gamma} \hat{\delta}} & =\frac{i}{12} \eta_{A}^{\hat{\epsilon}} \partial_{\hat{\epsilon}(\hat{\alpha}} \psi_{\hat{\beta} \hat{\gamma} \hat{\delta})}^{A} .
\end{aligned}
$$

In particular, the fact that these relations only involve the fields $\phi^{A B C D}, \lambda_{\hat{\alpha}}^{A B C}, h_{\hat{\alpha} \hat{\beta}}^{A B}, \psi_{\hat{\alpha} \hat{\beta} \hat{\gamma}}^{A}$ and $R_{\hat{\alpha} \hat{\beta} \hat{\gamma} \hat{\delta}}$ implies that these fields form a representation of the $O S p\left(8^{*} \mid 8\right)$ superalgebra without having to add any extra degrees of freedom. This is in agreement with the oscillator analysis of the previous sections.

\subsection{Dimensional reduction of six-dimensional conformal supermultiplets down to four dimensions}

We now consider the dimensional reduction of six-dimensional $(2,0)$ conformal doubleton supermultiplet of $O S p\left(8^{*} \mid 4\right)$ to four dimensions. It is described by the supermultiplet of coherent states

$$
\left|\phi^{[A B] \mid}(x)\right\rangle \oplus\left|\lambda_{\hat{\alpha}}^{A}(x)\right\rangle \oplus\left|h_{(\hat{\alpha} \hat{\beta})}(x)\right\rangle,
$$

where $A, B, \cdots$ denote $U S p(4)$ indices and $\hat{\alpha}, \hat{\beta}, \cdots=1,2,3,4$ denote the spinor indices of $S U^{*}(4)$. The spinor representation of $S U^{*}(4)$ decomposes with respect to the fourdimensional Lorentz group $S L(2, \mathbb{C})$ as

$$
\mathbf{4}=\left(\frac{1}{2}, 0\right) \oplus\left(0, \frac{1}{2}\right)
$$

this is the same as the decomposition of the fundamental representation of $S U(4)$ with respect to the compact subgroup $S U(2) \times S U(2)$. Thus we have the decomposition

$$
\left|h_{(\hat{\alpha} \hat{\beta})}(x)\right\rangle=\left|h_{(\alpha \beta)}(x)\right\rangle \oplus\left|h_{\alpha \dot{\beta}}(x)\right\rangle \oplus\left|h_{(\dot{\alpha} \dot{\beta})}(x)\right\rangle,
$$

where the coordinate vector $x_{\mu}$ is restricted to the four-dimensional Minkowski space. Comparing with the doubleton supermultiplet of $S U(2,2 \mid 4)[3]$ we see that $\left|h_{(\alpha \beta)}(x)\right\rangle$ and $\left|h_{(\dot{\alpha} \dot{\beta})}(x)\right\rangle$ are simply the self-dual and anti-self-dual components of the field strength of a 
vector field in four dimensions. The coherent state $\left|h_{(\alpha \dot{\beta})}(x)\right\rangle$ is the derivative of a scalar field

$$
\left|h_{\alpha \dot{\beta}}(x)\right\rangle=\partial_{\alpha \dot{\beta}}\left|\phi^{0}(x)\right\rangle
$$

The spinorial coherent state decomposes simply as

$$
\left|\lambda_{\hat{\alpha}}^{A}(x)\right\rangle=\left|\lambda_{\alpha}^{A}(x)\right\rangle \oplus\left|\lambda_{\dot{\alpha}}^{A}(x)\right\rangle
$$

The scalar coherent states $\left|\phi^{[A B] \mid}(x)\right\rangle$ descend directly to four dimensions. The resulting four-dimensional supermultiplet is in fact the $\mathcal{N}=4$ Yang-Mills supermultiplet with 6 scalar fields, 4 spin- $\frac{1}{2}$ fields and a vector field, and corresponds simply to the doubleton (minimal) unitary supermultiplet of $P S U(2,2 \mid 4)$. The $R$-symmetry group $U S p(4)$ of sixdimensional supermultiplet gets extended to $S U(4)$ under which $5+1$ scalars transform in the antisymmetric tensor representation. Four six-dimensional spinors of $S U^{*}(4)$ decompose into 4 chiral and 4 anti-chiral $S L(2, \mathbb{C})$ spinors in four dimensions, which also transform in $\mathbf{4}$ and $\overline{\mathbf{4}}$ representations of the four-dimensional $R$-symmetry $S U(4)$.

\subsection{Dimensional reduction of six-dimensional $(4,0)$ supermultiplet}

The fields of the $(4,0)$ doubleton supermultiplet of the six-dimensional superconformal group $O S p\left(8^{*} \mid 8\right)$ were listed in Table 5. Let us now discuss the dimensional reduction of this conformal supermultiplet using coherent state formalism.

The coherent state corresponding to the generalized graviton field strength decomposes as follows under dimensional reduction to four dimensions:

$$
\left|R_{(\hat{\alpha} \hat{\beta} \hat{\gamma} \hat{\delta})}(x)\right\rangle=\left|R_{(\alpha \beta \gamma \delta)}(x)\right\rangle \oplus\left|R_{(\dot{\alpha} \dot{\beta} \dot{\gamma} \dot{\delta})}(x)\right\rangle \oplus\left|R_{(\alpha \beta \gamma) \dot{\delta}}(x)\right\rangle \oplus\left|R_{(\dot{\alpha} \dot{\beta} \dot{\gamma}) \delta}(x)\right\rangle \oplus\left|R_{(\alpha \beta)(\dot{\gamma} \dot{\delta})}(x)\right\rangle
$$

The coherent states $\left|R_{(\dot{\alpha} \dot{\beta} \dot{\gamma} \dot{\delta})}(x)\right\rangle$ and $\left|R_{(\alpha \beta \gamma \delta)}(x)\right\rangle$ correspond to the chiral and anti-chiral components of the field strength of the four-dimensional graviton.

\begin{tabular}{|c|c|}
\hline $6 D$ Field & $4 D$ Decomposition \\
\hline$R_{(\hat{\alpha} \hat{\beta} \hat{\gamma} \hat{\delta})}$ & $R_{(\alpha \beta \gamma \delta)} \oplus R_{(\dot{\alpha} \dot{\beta} \dot{\gamma} \dot{\delta})} \oplus \partial_{\dot{\delta}(\gamma} h_{\alpha \beta)}^{0} \oplus \partial_{\delta(\dot{\gamma}} h_{\dot{\alpha} \dot{\beta})}^{0} \oplus \partial_{\alpha(\dot{\gamma}} \partial_{\dot{\delta}) \beta} \phi^{0}$ \\
\hline$\psi_{(\hat{\alpha} \hat{\beta} \hat{\gamma})}^{A}$ & $\psi_{(\alpha \beta \gamma)}^{A} \oplus \psi_{(\dot{\alpha} \dot{\beta} \dot{\gamma})}^{A} \oplus \partial_{\dot{\gamma}(\alpha} \lambda_{\beta)}^{A} \oplus \partial_{\alpha(\dot{\beta}} \lambda_{\dot{\gamma})}^{A}$ \\
\hline$h_{\hat{\alpha} \hat{\beta}}^{[A B] \mid}$ & $h_{\alpha \beta}^{[A B] \mid} \oplus h_{\dot{\alpha} \dot{\beta}}^{[A B] \mid} \oplus \partial_{\alpha \dot{\beta}} \phi^{[A B] \mid}$ \\
\hline$\lambda_{\hat{\alpha}}^{[A B C] \mid}$ & $\lambda_{\alpha}^{[A B C] \mid} \oplus \lambda_{\dot{\alpha}}^{[A B C] \mid}$ \\
\hline$\phi^{[A B C D] \mid}$ & $\phi^{[A B C D] \mid}$ \\
\hline
\end{tabular}

Table 6: Reduction to four dimensions of the fields of the $(4,0)$ multiplet. 
The coherent states $\left|R_{(\dot{\alpha} \dot{\beta} \dot{\gamma}) \delta}(x)\right\rangle$ and $\left|R_{(\alpha \beta \gamma) \dot{\delta}}(x)\right\rangle$ are descendants of the coherent states corresponding to self-dual and anti-self-dual components of the field strength of a fourdimensional vector field. The coherent state $\left|R_{(\alpha \beta)(\dot{\gamma} \dot{\delta})}(x)\right\rangle$ corresponds to the descendant of a scalar field. The other fields are reduced to four dimension with the same procedure and listed in Table 6 . The primary conformal fields in four dimension coincide precisely with the fields of the CPT-self-conjugate doubleton supermultiplet constructed in [28] which we reviewed in Section 2.2.

\subsection{Gauge potentials of the $(4,0)$ supermultiplet}

The components of supermultiplets obtained through the oscillator method are the dynamical gauge invariant degrees of freedom of the underlying theory. In particular, the oscillator method yields covariant field strengths and not gauge potentials.

For example, the $\mathcal{N}=4$ super-Yang-Mills supermultiplet obtained through this method contains the states transforming in the $(1,0) \oplus(0,1)$ representation of the Lorentz group [3]. In spinor notation, they are represented by two-index symmetric tensors, $F_{\alpha \beta}$ and $F_{\dot{\alpha} \dot{\beta}}$, each of them transforming irreducibly the Lorentz group. By contracting with the appropriate products of Pauli matrices they may be transformed to self-dual and antiself-dual antisymmetric tensors carrying vector indices:

$$
\frac{1}{2}\left(F_{\mu \nu}+* F_{\mu \nu}\right)=F_{\alpha \beta} \sigma_{\mu \nu}^{\alpha \beta} .
$$

Here $F_{\mu \nu}$ is the field strength of a Yang-Mills potential, $F_{\mu \nu}=\partial_{\mu} A_{\nu}-\partial_{\nu} A_{\mu}$ and $\sigma_{\mu \nu}$ is one of the diagonal $2 \times 2$ blocks of Lorentz generators in the spinor representation.

In this subsection we shall construct gauge potentials whose field strengths are the components of the $(4,0)$ supermultiplet given above. Since field strengths are obtained by taking derivatives of gauge potentials with respect to spacetime coordinates, it is standard practice to convert the field strengths given in terms of spinorial indices into vectorial indices by contraction with products of generalized Pauli matrices in six dimensions (i.e. the off-diagonal blocks of the six-dimensional Dirac matrices in the Weyl representation). For the $(2,0)$ doubleton supermultiplet this conversion was given already in [4]. For the symmetric tensor field $h_{(\alpha \beta)}$ one finds that it is associated to a two-form gauge field whose field strength is self-dual

$$
h_{M N P}=\partial_{[M} b_{N P]}=* h_{M N P} .
$$

Since a vector in six dimensions can also be described by a bispinor, one can represent the two form field $b_{M N}$ that transforms in the adjoint representation of $S U^{*}(4)$ by a field $b_{\hat{\beta}}^{\hat{\alpha}}$ with one upper and one lower spinor index subject to $b_{\hat{\alpha}}^{\hat{\alpha}}=0$. Then the field strength is

$$
h_{\hat{\alpha} \hat{\beta}}=h_{(\hat{\alpha} \hat{\beta})}=\partial_{\hat{\gamma}(\hat{\alpha}} b_{\hat{\beta})}^{\hat{\gamma}}
$$


and the gauge parameter is a vector $\chi^{[\hat{\alpha} \hat{\beta}]}$ of $S U^{*}(4)$. The gauge potential for the generalized graviton field strength $R_{\hat{\alpha} \hat{\beta} \hat{\gamma} \hat{\delta}}$ is a tensor $C_{(\hat{\beta} \hat{\gamma} \hat{\delta})}^{\hat{\alpha}}$, carrying fundamental indices of $S U^{*}(4)$, subject to the tracelessness condition

$$
C_{\hat{\alpha} \hat{\beta} \hat{\gamma}}^{\hat{\gamma}}=0
$$

The corresponding gauge transformation of this potential is

$$
C_{\hat{\beta} \hat{\gamma} \hat{\delta}}^{\hat{\alpha}} \rightarrow C_{\hat{\beta} \hat{\gamma} \hat{\delta}}^{\hat{\alpha}}+\partial_{\hat{\omega}(\hat{\beta}} \chi_{\hat{\gamma} \hat{\delta})}^{\hat{\omega} \hat{\alpha}}
$$

with the gauge parameter $\chi_{(\hat{\gamma} \hat{\delta})}^{[\hat{\alpha} \hat{\beta}]}$ subject to the tracelessness condition,

$$
\chi_{\hat{\beta} \hat{\gamma}}^{\hat{\alpha} \hat{\gamma}}=0
$$

Similarly, the gauge potential corresponding to the generalized gravitino field strength is a tensor $\psi_{(\hat{\beta} \hat{\gamma})}^{\hat{\alpha}}$ which is also traceless,

$$
\psi_{\hat{\alpha} \hat{\beta}}^{\hat{\beta}}=0
$$

and transforms under a gauge transformation as

$$
\psi_{\hat{\beta} \hat{\gamma}}^{\hat{\alpha}} \rightarrow \psi_{\hat{\beta} \hat{\gamma}}^{\hat{\alpha}}+\partial_{\hat{\omega}(\hat{\beta}} \chi_{\hat{\gamma})}^{\hat{\omega} \hat{\alpha}}
$$

the gauge parameter $\chi_{\hat{\gamma}}^{[\hat{\alpha} \hat{\beta}]}$ satisfies the condition $\chi_{\hat{\beta}}^{\hat{\alpha} \hat{\beta}}=0$. It is important to note that the local gauge symmetry of the generalized gravitino field is distinct from supersymmetry, as it can be inferred from the fact that the gauge parameter $\chi_{\hat{\gamma}}^{\hat{\alpha} \hat{\beta}}$ does not transform in the fundamental representation of $S U^{*}(4)$.

In Table 7 , we list the gauge fields corresponding to the $(4,0)$ supermultiplet and their gauge parameters in both vectorial as well as spinorial notation of the six-dimensional Lorentz group $S U^{*}(4)$. The gauge potentials in vector notation agree with the results of $[40,41]$, with the exception of the potential $C$, which in our case is related to the generalized Riemann tensor by only one derivative.

By examining the gauge potentials written in the vectorial notation, it is clear that the $(4,0)$ theory contains "higher-spin" gauge fields in the sense that the generalized graviton field $C_{M N O, P Q}$ has six-dimensional vector indices with mixed symmetry. Theories of this sort were studied in $[70,71]$ and are the object of several no-go theorems in the literature. Constraints on an interacting $(4,0)$ theory from such higher-spin no-go theorems will be discussed in Section 5.1.

We have seen in the previous section that the fields of the $(4,0)$ supermultiplet reduce to supermultiplets in five and four dimensions that do not have any fields with spin greater than two. In particular, in six dimensions we have two different fields that reduce to 


\begin{tabular}{|c|c|c|c|c|}
\hline$S O(4)$ rep & Vectorial field & Constraints & $S U^{*}(4)$ rep & Spinorial field \\
\hline \multirow[t]{2}{*}{$(2,0)$} & $R_{[M N O][P Q R]}$ & $\begin{array}{c}* R=R *=R \\
R_{M N O P Q R}=R_{P Q R M N O}\end{array}$ & & $R_{(\hat{\alpha} \hat{\beta} \hat{\gamma} \hat{\delta})}=\partial_{\hat{\lambda}(\hat{\alpha}} C_{\hat{\beta} \hat{\gamma} \hat{\delta})}^{\hat{\lambda}}$ \\
\hline & $C_{[M N O][P Q]}$ & $\begin{array}{c}* C=C \\
C_{[M N O P] Q}=0\end{array}$ & & $C_{(\hat{\beta} \hat{\gamma} \hat{\gamma})}^{\hat{\alpha}}, \quad C_{\hat{\alpha} \hat{\beta} \hat{\gamma}}^{\hat{\gamma}}=0$ \\
\hline$\left(\frac{3}{2}, 0\right)$ & $\psi_{[M N O] \hat{\alpha}}$ & $* \psi=\psi$ & & $\psi_{(\hat{\alpha} \hat{\beta} \hat{\gamma})}=\partial_{\hat{\lambda}(\hat{\alpha}} \psi_{\hat{\beta} \hat{\gamma})}^{\hat{\lambda}}$ \\
\hline & $\psi_{[M N] \hat{\alpha}}$ & & & $\psi_{(\hat{\beta} \hat{\gamma})}^{\hat{\alpha}}, \quad \psi_{\hat{\alpha} \hat{\beta}}^{\hat{\beta}}=0$ \\
\hline$(1,0)$ & $h_{[M N P]}$ & $* h=h$ & & $h_{(\hat{\alpha} \hat{\beta})}=\partial_{\hat{\gamma}(\hat{\alpha}} b_{\hat{\beta})}^{\hat{\gamma}}$ \\
\hline & $b_{[M N]}$ & & & $b_{\hat{\beta}}^{\hat{\alpha}}, \quad b_{\hat{\alpha}}^{\hat{\alpha}}=0$ \\
\hline$\left(\frac{1}{2}, 0\right)$ & $\lambda_{\hat{\alpha}}$ & & $\square$ & $\lambda_{\hat{\alpha}}$ \\
\hline$(0,0)$ & $\phi$ & & 1 & $\phi$ \\
\hline
\end{tabular}

Table 7: Correspondence between fields of the $(4,0)$ supermultiplet with vectorial indices and spinorial indices. Fields are organized according to the corresponding representations of the little group $S O(4)=S U(2) \times S U(2)$. Lower and higher spinor indices correspond to fundamental and anti-fundamental $\mathbf{4}$ and $\overline{\mathbf{4}}$ indices respectively.

the massless graviton in five and four dimensions, namely the generalized graviton $C$ and the metric graviton $g$ of Poincaré supergravity, just like the four-dimensional $\mathcal{N}=4$ Yang-Mills supermultiplet can be obtained from the conformal $(2,0)$ theory as well as from the non-conformal $(1,1)$ super-Yang-Mills theory in six dimensions. This is a special feature of the $(4,0)$ multiplet which is not present in a generic higher-spin theory in six dimensions.

\subsection{The (4,0) supergravity multiplet as the square of the $(2,0)$ multiplet}

The degrees of freedom of the $(4,0)$ supermultiplet constructed in the previous section can be brought into direct correspondence with the states obtained from the direct product of two six-dimensional $(2,0)$ multiplets. ${ }^{17}$ In order to do so we need to consider the transformation properties of the states of the $(4,0)$ multiplet under an $U S p(4) \times U S p(4)$ subgroup of the $U S p(8) R$-symmetry group of the theory.

\footnotetext{
${ }^{17}$ This is similar to the fact that the fields in the tensor product of two $(1,1)$ vector multiplets are in one to one correspondence with the fields of the $(2,2)$ Poincaré graviton multiplet.
} 


\begin{tabular}{|c|c|c|}
\hline Field & Decomposition & $U S p(4) \times U S p(4)$ representations \\
\hline$\phi^{[A B C D] \mid}$ & $\phi^{[a b]|[\check{a} \breve{b}]|} \oplus \phi^{a \check{a}} \oplus \phi$ & $(\mathbf{5}, \mathbf{5}) \oplus(\mathbf{4}, \mathbf{4}) \oplus(1,1)$ \\
\hline$\lambda_{\hat{\alpha}}^{[A B C] \mid}$ & $\lambda_{\hat{\alpha}}^{\check{a}[a b] \mid} \oplus \lambda_{\hat{\alpha}}^{a[\check{a} b] \mid} \oplus \lambda_{\hat{\alpha}}^{a} \oplus \lambda_{\hat{\alpha}}^{\check{a}}$ & $(\mathbf{5}, \mathbf{4}) \oplus(\mathbf{4}, \mathbf{5}) \oplus(\mathbf{4}, 1) \oplus(1, \mathbf{4})$ \\
\hline$h_{(\hat{\alpha} \hat{\beta})}^{[A B] \mid}$ & $h_{(\hat{\alpha} \hat{\beta})}^{[a b] \mid} \oplus h_{(\hat{\alpha} \hat{\beta})}^{[a \check{b}] \mid} \oplus h_{(\hat{\alpha} \hat{\beta})}^{a \check{a}} \oplus h_{(\hat{\alpha} \hat{\beta})}$ & $(\mathbf{5}, 1) \oplus(1, \mathbf{5}) \oplus(\mathbf{4}, \mathbf{4}) \oplus(1,1)$ \\
\hline$\psi_{(\hat{\alpha} \hat{\beta} \hat{\gamma})}^{A}$ & $\psi_{(\hat{\alpha} \hat{\beta} \hat{\gamma})}^{a} \oplus \psi_{(\hat{\alpha} \hat{\beta} \hat{\gamma})}^{\check{a}}$ & $(\mathbf{4}, 1) \oplus(1, \mathbf{4})$ \\
\hline$R_{(\hat{\alpha} \hat{\beta} \hat{\gamma} \hat{\delta})}$ & $R_{(\hat{\alpha} \hat{\beta} \hat{\gamma} \hat{\delta})}$ & $(1,1)$ \\
\hline
\end{tabular}

Table 8: Decomposition of the component fields of the $(4,0)$ supergravity multiplet in irreducible representations of the $U S p(4) \times U S p(4)$ subgroup of $U S p(8)$.

\begin{tabular}{|c|c|c|c|}
\hline & $h_{\hat{\gamma} \hat{\delta}}$ & $\lambda_{\hat{\gamma}}^{a}$ & $\phi^{[\check{a} \bar{b}]}$ \\
\hline$h_{\hat{\alpha} \hat{\beta}}$ & $R_{\hat{\alpha} \hat{\beta} \hat{\gamma} \hat{\delta}} \oplus \partial_{\hat{\alpha}(\hat{\gamma}} h_{\hat{\delta}) \hat{\beta}} \oplus \partial_{\hat{\alpha}(\hat{\gamma}} \partial_{\hat{\delta}) \hat{\beta}} \phi^{0}$ & $\psi_{\hat{\alpha} \hat{\beta} \hat{\gamma}}^{\check{a}} \oplus \partial_{\hat{\gamma}(\hat{\alpha}} \lambda_{\hat{\beta})}^{\check{a}}$ & $h_{\hat{\alpha} \hat{\beta}}^{[a \check{b}] \mid}$ \\
\hline$\lambda_{\hat{\alpha}}^{a}$ & $\psi_{\hat{\alpha} \hat{\gamma} \hat{\delta}}^{a} \oplus \partial_{\hat{\alpha}(\hat{\gamma}} \lambda_{\hat{\delta})}^{a}$ & $h_{\hat{\alpha} \hat{\gamma}}^{a \check{\gamma}} \oplus \partial_{\hat{\alpha} \hat{\gamma}} \phi^{a \check{a}}$ & $\lambda_{\hat{\alpha}}^{a[\widetilde{a} \breve{b}]}$ \\
\hline$\phi^{[a b] \mid}$ & $h_{\hat{\gamma} \hat{\delta}}^{[a b] \mid}$ & $\lambda_{\hat{\gamma}}^{\breve{a}[a b] \mid}$ & $\phi^{[a b] \mid[\check{a} \breve{b}]}$ \\
\hline
\end{tabular}

Table 9: The field content of the $(4,0)$ multiplet is obtained taking the direct product of two $(2,0)$ multiplets. Each one of the three rows and columns correspond to a different component field of the two $(2,0)$ multiplets. Each of the nine entries correspond to the decomposition of the product of two fields in irreducible representations of $S U^{*}(4)$ and $U S p(4) \times U S p(4)$.

The fundamental representation of $U S p(8)$ decomposes as

$$
8=(4,1) \oplus(\mathbf{1}, \mathbf{4}) .
$$

We will denote as $a, b=1, \ldots, 4$ and $\check{a}, \check{b}=1, \ldots, 4$ the fundamental indices of the two $U S p(4)$ groups, so that an $U S p(8)$ index $A=1, \ldots, 8$ is represented by the pair $A=(a, \breve{a})$. The $U S p(8)$ symplectic matrix $\Omega$ can be brought to a block-diagonal form so that $\Omega_{a \check{a}}=\Omega^{a \check{a}}=0$. The various component fields of the multiplet decompose in irreducible representations of $U S p(4) \times U S p(4)$. This decomposition is listed in Table 8 . To obtain the $(4,0)$ multiplet, we now need to take the direct product of two copies of the $(2,0)$ multiplet each transforming under a different $U S p(4)$ subgroup of the $U S p(8)$ $R$-symmetry group. As we have seen, each $(2,0)$ multiplet has three component fields.

In each of the nine entries of Table 9 we decompose a different product of two component fields in irreducible representations of $S U^{*}(4)$. The states obtained in this way are labeled by $U S p(4) \times U S p(4)$ indices and exactly match the field content of the $(4,0)$ multiplet as listed in Table 8 . 


\section{Interacting $(2,0)$ superconformal theory and $(4,0)$ theory}

It is generally believed that $A d S / C F T$ duality between IIB superstring theory on $A d S_{5} \times$ $S^{5}$ and $\mathcal{N}=4$ super Yang-Mills theory in four dimensions has a higher-dimensional counterpart, relating M-theory on $A d S_{7} \times S^{4}$ and an interacting $(2,0)$ superconformal theory in six dimensions. The six-dimensional $(2,0)$ conformal supermultiplet, which was called the doubleton supermultiplet, appears as gauge modes in the compactification of 11-dimensional supergravity over $S^{4}$ and decouples from the Kaluza-Klein spectrum. However the entire Kaluza-Klein tower of 11 dimensional supergravity over $S^{4}$ can be obtained by tensoring of the $(2,0)$ doubleton supermultiplets and fitted into massless and massive supermultiplets of $O S p\left(8^{*} \mid 4\right)$ [4]. The $(2,0)$ supermultiplet does not have a Poincaré limit as a representation of the $A d S_{7}$ supergroup $O S p\left(8^{*} \mid 4\right)$ and it was pointed out in [4] that the theory based on the $(2,0)$ doubleton supermultiplet must be a superconformal field theory that lives on the boundary of $A d S_{7}$ which is the six-dimensional Minkowski space. The $(2,0)$ superconformal multiplet consists of 5 scalars, four symplectic Majorana-Weyl spinors, and one two-form gauge field whose field strength is subject to six-dimensional self-duality. Due to self-duality, a local covariant free action for the two-form gauge field vanishes identically. Hence one can have only covariant equations of motion for the free $(2,0)$ theory.

Using $\mathrm{M} /$ superstring theory arguments, Witten showed that there must exist interacting six-dimensional superconformal theories based on the $(2,0)$ doubleton supermultiplet [38]. These theories are classified by the Dynkin diagrams of simply laced groups (A-DE) and describe the low energy decoupling limits of IIB superstrings on a $K 3$ manifold. Among these theories those belonging to the A-series also have M-theory description in terms of $M 5$ branes [38]. Witten has argued that these interacting superconformal $(2,0)$ theories may exist only as quantum theories without a classical description in terms of invariant actions and/or covariant equations of motion [72]. The reduction of the $(2,0)$ theory on a two torus yields the $\mathcal{N}=4$ super Yang-Mills theory in four dimensions. Re-

ducing the interacting $(2,0)$ theory on Riemann surfaces with punctures leads to a plethora of $\mathcal{N}=2$ superconformal field theories in four dimensions [73] whose five-dimensional supergravity duals were studied in [74]. These recent results lend further support for the existence of interacting $(2,0)$ superconformal theories in six dimensions.

The $(4,0)$ doubleton supermultiplet of $O S p\left(8^{*} \mid 8\right)$ we studied above can be decomposed into supermultiplets of $O S p\left(8^{*} \mid 4\right)$ subalgebra. Denoting the $U S p(4)$ indices as $a, b, c=1,2,3,4$ we find that it contains one generalized graviton field strength $(2,0)$ supermultiplet consisting of the fields

$$
R_{(\hat{\alpha} \hat{\beta} \hat{\gamma} \hat{\delta})}(x), \quad \psi_{(\hat{\alpha} \hat{\beta} \hat{\gamma})}^{a}(x), \quad h_{(\hat{\alpha} \hat{\beta})}^{[a b] \mid}(x) \oplus h_{(\hat{\alpha} \hat{\beta})}(x), \quad \lambda_{\hat{\alpha}}^{[a b c]}(x), \quad \phi(x)
$$


four $(2,0)$ generalized gravitino supermultiplets consisting of the fields

$$
\psi_{(\hat{\alpha} \hat{\beta} \hat{\gamma})}(x), \quad h_{(\hat{\alpha} \hat{\beta})}^{a}(x), \quad \lambda_{\hat{\alpha}}^{[a b] \mid}(x) \oplus \lambda_{\hat{\alpha}}(x), \quad \phi^{[a b c] \mid}(x)
$$

and five $(2,0)$ doubleton supermultiplets containing the fields

$$
h_{(\hat{\alpha} \hat{\beta})}(x), \quad \lambda_{\hat{\alpha}}^{a}(x), \quad \phi^{[a b] \mid}(x)
$$

Hence we expect any interacting theory of $(4,0)$ supermultiplet to have a consistent truncation to an interacting theory describing the coupling of a generalized $(2,0)$ graviton supermultiplet to five $(2,0)$ doubleton supermultiplets. If this interacting theory admits a limit where the $(2,0)$ graviton supermultiplet decouples one is then left with a theory of five $(2,0)$ doubleton supermultiplets.

The corresponding decomposition of the four dimensional doubleton supermultiplet of $S U(2,2 \mid 8)$ with respect to $P S U(2,2 \mid 4)$ leads to $\mathcal{N}=4$ Weyl multiplet plus its CPT conjugate, four chiral $\mathcal{N}=4$ gravitino supermultiplets and their CPT conjugates, and six Yang-Mills supermultiplets. There exists twistor string theories in four dimensions

$[75,76]$ which are invariant under $\operatorname{PSU}(2,2 \mid 4)$ and, at least in principle, can be used to calculate scattering amplitudes of $\mathcal{N}=4$ super Yang-Mills theory. One finds that twistor string description requires the introduction of $\mathcal{N}=4$ graviton supermultiplets coupled conformally to Yang-Mills supermultiplets [77]. The helicity of the conformal graviton that appears in scattering amplitude calculations is correlated with the helicity of the Yang-Mills gluons [78]. Since $\mathcal{N}=4$ super-Yang-Mills theory is parity invariant, both the positive and negative helicity conformal graviton supermultiplets must be present in the resulting theory.

Since supertwistors exist in six dimensions it is natural to expects that there should exist a six-dimensional analog of either Witten's or Berkovits' twistor string theory, which can be used to calculate amplitudes of an interacting $(2,0)$ "gauge" theory and, similarly to their four-dimensional analogs, of the six-dimensional $(2,0)$ conformal graviton supermultiplets. The unique candidate for such a conformal graviton supermultiplet is the one given in eq. (5.1). Under reduction to four dimensions one then obtains a CPT invariant theory describing the coupling of $\mathcal{N}=4$ super-Yang-Mills theory to conformal supergravity.

\subsection{Perspectives from Weinberg and Coleman-Mandula theorems}

As briefly mentioned in Section 4.6, the gauge potentials in the $(4,0)$ supermultiplet can be regarded as massless higher-spin fields with space-time indices of mixed symmetry. Such theories have been studied at length in the literature and are expected to obey constraints following from higher-spin gauge invariance. In asymptotically flat space-times, several nogo theorems - most notably Weinberg's theorem [79] and the Coleman-Mandula theorem 
[80] - rule out possible interactions between fields of higher and lower spin under certain assumptions.

The original Weinberg theorem showed that particles with spin greater than two cannot mediate long-distance interactions in a flat four-dimensional space-time [79]. Specifically, the theorem considers the scattering of one higher-spin field $\varphi_{\mu_{1} \ldots \mu_{S}}(q)$ with $n-1$ scalars. In the soft limit, in which the momentum $q$ of the higher-spin field is small, the scattering amplitude behaves as

$$
A_{n}\left(\phi_{1}, \ldots, \phi_{n-1}, \varphi_{\mu_{1} \ldots \mu_{S}}\right)=A_{n-1}\left(\phi_{1}, \ldots, \phi_{n-1}\right) \sum_{i=1}^{n-1} g_{i} \frac{p_{i}^{\mu_{1}} \ldots p_{i}^{\mu_{S}} \epsilon_{\mu_{i} \ldots \mu_{S}}(q)}{2 p_{i} \cdot q}
$$

where $p_{i}$ are the momenta of the scalars fields, $\epsilon_{\mu_{1} \ldots \mu_{S}}$ is the polarization tensor for the higher-spin field and $A_{n-1}$ is a scattering amplitude involving only $n-1$ scalars. An higher-spin gauge transformation acts on the polarization tensor as

$$
\epsilon(q) \rightarrow \epsilon(q)+i q \Lambda(q) .
$$

If there are no extra factors of $q$ present in the cubic vertices, a gauge transformation of the external higher-spin field could change the amplitude by a finite amount due to a cancellation of the poles in $p_{i} \cdot q$. Hence, in order to decouple the unphysical states and preserve gauge invariance in this limit, one needs to impose an extra condition on the momenta to cancel these contributions:

$$
\sum_{i=1}^{n-1} g_{i} p_{i, \mu_{1}} \ldots p_{i, \mu_{S-1}}=0
$$

where $g_{i}$ are the coupling constants corresponding to the $\varphi \phi \phi$ vertices. Unless $S=1$ or $S=2$, this condition is too restrictive and the $g_{i}$ are forced to vanish altogether. The theorem can be further generalized to consider the scattering of fields of different spin [82]. In this case, the problem arises only when the field exchanged has spin lower than the external higher-spin field. It is important to point out that Weinberg's theorem does not rule out all interactions in a theory with higher-spin fields. The argument applies only to amplitudes with a single massless higher-spin field, assumes that the theory is local and restricts only the minimal cubic couplings of the higher-spin field with the other fields of the theory $[81,82]$. Extensions of Weinberg's theorem by Witten [83] and, more recently, by Porrati [84] also imply that higher-spin fields cannot be minimally coupled to gravity in a consistent manner in flat spacetimes.

Coleman-Mandula theorem [80] and its extension by Haag, Lopuszanski and Sohnius [85] state that the maximal extension of Poincare symmetry algebra is the semi-direct sum of a Poincaré (or conformal) superalgebra and a purely internal Lie algebra whose generators commute with those of the Poincaré (or superconformal) algebra. The main assumptions of Haag, Lopuszanski and Sohnius are that the S-matrix for elastic two-body 
scattering is nontrivial at all angles and that there are finitely many particles types below any given energy.

Despite the no-go theorems in the literature, in recent years many novel results were obtained showing that it is possible to have consistent interactions of higher-spin fields with lower spin-fields (including gravity) if one relaxes some of the assumptions. Interacting higher-spin theories in asymptotically flat spacetimes were successfully constructed using a lightcone gauge approach [86, 87, 88, 89] or techniques based on Noether's procedure $[90,91,92]$ (see [93] for a comprehensive review).

In case of a putative interacting theory of the $(4,0)$ supermultiplet, both the higher-spin no-go theorems and the examples of higher-spin theories in the literature give interesting hints on the nature of the allowed interactions. Weinberg's theorem appears to exclude minimal cubic vertices involving the higher-spin field of the theory - the generalized graviton $C_{\beta \gamma \delta}^{\alpha}$ - and two fields of lower spin. In fact, contributions from such vertices in the soft-limit $q \rightarrow 0$ are already excluded by the mixed symmetry of the higher-spin field's polarization tensor in (5.4), which has at least two antisymmetric Lorentz indices.

The fact that many known examples of consistent higher-spin theories involve nonlocal interactions suggests that the interactions of the $(4,0)$ theory may also be non-local. Interestingly, interactions of this sort may be likely to appear in the $(2,0)$ theory. The authors of $[94,95]$ have looked for possible three-point and four-points amplitudes in the $(2,0)$ theory which were rational in the kinematic invariants $s_{i j}=-\left(k_{i}+k_{j}\right)^{2}$ and found that, to write down interactions consistent with the superconformal symmetry, one may need to give up either six-dimensional Lorentz covariance [95] or to consider amplitudes which are not rational in $s_{i j}$.

At this stage, further study is required to determine whether it is possible to introduce consistent interactions in a theory of the $(4,0)$ multiplet. In particular, it would be interesting to study candidate cubic vertices in the light-cone formalism of [88] or using the techniques of [91, 92]. As mentioned in the previous section, the study of six-dimensional twistor strings could also offer some insight on an interacting $(4,0)$ theory.

\section{Discussion}

Maximal ungauged Poincaré supergravity in six dimensions has $(2,2)$ supersymmetry with $R$-symmetry group $U S p(4) \times U S p(4)$. Its $\mathrm{U}$-duality group is $S O(5,5)$ with maximal compact subgroup $U S p(4) \times U S p(4)[96]$. The theory contains 5 antisymmetric tensor fields and 16 vector fields. The field strengths of the tensor fields together with their duals form a 10-dimensional representation of the $U$-duality group $S O(5,5)$, and the vector fields transform in the spinor representation.

The equations of motion of linearized maximal supergravity in six dimensions are not conformally invariant. This is best seen by the fact that the $(2,2)$ graviton supermul- 
tiplet contains field strengths whose transformation properties under the Lorentz group $S U^{*}(4)$ are described by Young tableaux which have more than one row. In contrast, field strengths of massless conformal fields in six dimensions have $S U^{*}(4)$ tableaux that contain only one row.

For the same reason, the maximally $(1,1)$ supersymmetric Yang-Mills theory cannot be conformally invariant in six dimensions. Nevertheless, the six-dimensional $(1,1)$ supersymmetric Yang-Mills theory reduces to $\mathcal{N}=4$ super Yang-Mills theory in four dimensions which is conformally invariant, not only classically but also at the quantum level.

At the same time, strong evidence suggests that there exists a conformally invariant interacting theory with fields in the $(2,0)$ doubleton supermultiplet of $O S p\left(8^{*} \mid 4\right)$ that is dual to $M$-theory on $A d S_{7} \times S^{4}$. It has been argued that this theory reduces to $\mathcal{N}=4$ super Yang-Mills in four dimensions as well [38, 39, 97, 98].

This raises the question whether maximal (ungauged) supergravity theories in six and four dimensions follow a similar pattern. That is, since the $(2,2)$ six-dimensional Poincaré supergravity reduces to the $\mathcal{N}=8$ supergravity in four dimensions and the linearized field equations of the latter are conformally invariant, it is interesting to ask whether there exists a maximal supergravity in six dimensions whose field equations at the linearized level are conformally invariant and whose fields can be identified with a conformal supermultiplet in six dimensions. The theory of the $(4,0)$ supermultiplet we constructed in this paper is indeed a likely candidate since this multiplet is a representation of the six-dimensional superconformal algebra and it reduces to the maximal supergravity multiplet in four dimensions. Moreover, in close similarity with the four-dimensional maximal gauge and supergravity multiplets, the six-dimensional $(4,0)$ and $(2,0)$ multiplets enjoy a KLT-like relation, the former being the tensor product of two copies of the latter. This $(4,0)$ supermultiplet would be the gravitational analog of the $(2,0)$ multiplet and, if an interacting non-metric theory based on it exists, then that theory may potentially be equivalent, at the linearized level, to the $(4,0)$ conformal gravity theory studied in $[40,41]$ with a different formalism. We should perhaps stress once more that the fundamental principles underlying the interacting $(2,0)$ theory are not yet known. Once these principles are uncovered we may be able to investigate the question whether the same principles can be extended to an interacting $(4,0)$ theory.

In the formalism of [40, 41], field strengths and gauge potentials of the $(4,0)$ supermultiplet are written with vector indices of mixed symmetry. If the linearized multiplet can be promoted to a fully interacting theory, we expect higher-spin no-go theorems present in the literature to restrict the allowed interactions. In particular, we would expect the mixed-symmetry field $C$ to be coupled non-minimally to the fields of lower spin.

Remarkably, the $S U^{*}(4)$ representations of the fields of six-dimensional $(4,0)$ supermultiplet remain irreducible with respect to the covering group, $U S p(2,2)$, of the fivedimensional Lorentz group $S O(4,1)$. Hence, under dimensional reduction to five dimen- 
sions the field content remains the same and, when restricted to the five-dimensional Lorentz group, the field content coincides with that of maximal supergravity in five dimensions. This is also consistent with the fact that $R$-symmetry group of maximal supergravity in five-dimensional is $U S p(8)$.

We do not expect that a non-metric maximal supergravity based on the $(4,0)$ supermultiplet admits a covariant action in six dimensions. However, as suggested for the six-dimensional interacting $(2,0)$ theory, one may try to gain some insights into the sixdimensional theory by studying its dimensional reduction to five-dimensions [97]. Since the $(4,0)$ supermultiplet reduces to the fields of $\mathcal{N}=8$ Poincaré supergravity, we expect the 42 scalars to parameterize the coset space

$$
\frac{E_{6(6)}}{U S p(8)}
$$

and the equations of motion of the presumed six-dimensional theory to have $E_{6(6) \text { global }} \times$ $U S p(8)_{l o c a l}$ symmetry. The self-dual tensor fields of the $(4,0)$ supermultiplet should then transform linearly in the $\mathbf{2 7}$ of $E_{6(6)}$.

The conformal superalgebras discussed in this paper are valuable tools in the study of possible counterterms for maximal supergravity in various dimensions. In oscillator language, linearized supersymmetric counterterms can be obtained acting with fermionic generators on a suitable lowest weight state. In general, states corresponding to half-BPS linearized counterterms with four fields can be obtained by starting with four copies of the Fock vacuum and acting with all sixteen grade +1 fermionic generators. Similarly, candidate non-BPS counterterms can be obtained acting with 32 odd generators on a given lowest-weight state, which needs to be a Lorentz scalar and a singlet under the $R$-symmetry group.

In four dimensions we obtain the half-BPS state

$$
4 D: \quad\left(L^{i x}\right)^{16}|0,0,0,0\rangle
$$

where the sixteen odd generators $L^{i x}$ are defined in Section 2.2. This state is manifestly invariant under 32 supersymmetry generators and, upon acting with the intertwiner operator, leads to the supersymmetric completion of the $R^{4}$ term which plays an important role in the analysis of candidate counterterms in four dimensions [30, 99, 100, 24, 101, 102]. Note that the action of the 16 generators above is equivalent to a superspace integral over half of the fermionic coordinates.

Similarly, in three dimensions one can obtain a simple half-BPS term with four fields from the state

$$
3 D: \quad\left(A^{i \kappa}\right)^{16}|0,0,0,0\rangle
$$

where $A^{i \kappa}$ is defined in Section 3.4. Three-dimensional $\mathcal{N}=16$ supergravity has a better UV behavior than four-dimensional $\mathcal{N}=8$ supergravity, and it is expected to be finite 
if the four-dimensional theory is finite. It would be interesting to see if invariance under $E_{8(8)}$ and $S O(16)$ pose constraints on candidate counterterms in three dimensions which are more stringent than the ones from $E_{7(7)}$ and $S U(8)$ in four dimensions.

In six dimensions it is possible to get a counterterm with four fields from the state

$$
6 D: \quad\left(A^{i x}\right)^{16}|0,0,0,0\rangle,
$$

where $A^{i x}$ is defined in Section 4.2. The four-graviton part of the six-dimensional counterterm contains four copies of the Weyl tensor $R_{\hat{\alpha} \hat{\beta} \hat{\gamma} \hat{\delta}}$ which belongs to the $(4,0)$ conformal supermultiplet, and can be written as,

$$
6 D: \quad R^{4}=\epsilon^{\hat{\alpha}_{1} \hat{\beta}_{1} \hat{\gamma}_{1} \hat{\delta}_{1}} \epsilon^{\hat{\alpha}_{2} \hat{\beta}_{2} \hat{\gamma}_{2} \hat{\delta}_{2}} \epsilon^{\hat{\alpha}_{3} \hat{\beta}_{3} \hat{\gamma}_{3} \hat{\delta}_{3}} \epsilon^{\hat{\alpha}_{4} \hat{\beta}_{4} \hat{\gamma}_{4} \hat{\delta}_{4}} R_{\hat{\alpha}_{1} \hat{\alpha}_{2} \hat{\alpha}_{3} \hat{\alpha}_{4}} R_{\hat{\beta}_{1} \hat{\beta}_{2} \hat{\beta}_{3} \hat{\beta}_{4}} R_{\hat{\gamma}_{1} \hat{\gamma}_{2} \hat{\gamma}_{3} \hat{\gamma}_{4}} R_{\hat{\delta}_{1} \hat{\delta}_{2} \hat{\delta}_{3} \hat{\delta}_{4}} .
$$

The symmetries of $R_{\hat{\alpha} \hat{\beta} \hat{\gamma} \hat{\delta}}$ forbid other index contractions, suggesting that the structure in equation (6.5) is unique. Under dimensional reduction to four dimensions it reduces to the standard CPT-invariant $R^{4}$ term of the form

$$
4 D: \quad R_{\alpha \beta \gamma \delta} R^{\alpha \beta \gamma \delta} R_{\dot{\alpha} \dot{\beta} \dot{\gamma} \dot{\delta}} R^{\dot{\alpha} \dot{\beta} \dot{\gamma} \dot{\delta}} .
$$

The uniqueness of the $R^{4}$ term in four dimensions lends further support to the expectation that the corresponding $R^{4}$ term in six dimension (6.5) is unique as well. Future work will investigate in detail the application of oscillator techniques to the study of BPS and non-BPS counterterms for maximal supergravity in various dimensions.

\section{Acknowledgements}

We would like to thank H. Elvang, Y.-t. Huang, C. Hull and H. Samtleben for discussions and the Kavli Institute for Theoretical Physics for hospitality during the course of this work. This research was supported in part by the National Science Foundation under grant PHY-08-55356 and in part by the National Science Foundation under grant PHY-05-51164. RR also acknowledges the support of the A.P. Sloan Foundation.

\section{A A Review of the Oscillator Construction of the Positive En- ergy Unitary Representations of the $4 D$ Conformal Group $S U(2,2)$}

The generators of the conformal group in four dimensions $S U(2,2)$ (the two sheeted covering of $S O(4,2))$ satisfy the commutation relations

$$
\left[M_{\mu \nu}, M_{\rho \sigma}\right]=i\left(\eta_{\nu \rho} M_{\mu \sigma}-\eta_{\mu \rho} M_{\nu \sigma}-\eta_{\nu \sigma} M_{\mu \rho}+\eta_{\mu \sigma} M_{\nu \rho}\right)
$$




$$
\begin{aligned}
{\left[P_{\mu}, M_{\rho \sigma}\right] } & =i\left(\eta_{\mu \rho} P_{\sigma}-\eta_{\mu \sigma} P_{\rho}\right) \\
{\left[K_{\mu}, M_{\rho \sigma}\right] } & =i\left(\eta_{\mu \rho} K_{\sigma}-\eta_{\mu \sigma} K_{\rho}\right) \\
{\left[D, M_{\mu \nu}\right] } & =\left[P_{\mu}, P_{\nu}\right]=\left[K_{\mu}, K_{\nu}\right]=0 \\
{\left[P_{\mu}, D\right] } & =i P_{\mu} ; \quad\left[K_{\mu}, D\right]=-i K_{\mu} \\
{\left[P_{\mu}, K_{\nu}\right] } & =2 i\left(\eta_{\mu \nu} D-M_{\mu \nu}\right)
\end{aligned}
$$

where $M_{\mu \nu}$ are the Lorentz group generators, $P_{\mu}$ the four-momentum generators, $D$ the dilatation generator and generators of $K_{\mu}$ the special conformal generators. The Lorentz metric is $\eta_{\mu \nu}=\operatorname{diag}(+,-,-,-)$ with $(\mu, \nu, \cdots=0,1,2,3)$

Defining

$$
M_{\mu 5}=\frac{1}{2}\left(P_{\mu}-K_{\mu}\right), \quad M_{\mu 6}=\frac{1}{2}\left(P_{\mu}+K_{\mu}\right), \quad M_{56}=-D,
$$

the Lie algebra of the conformal group can be written in a manifestly $S O(4,2)$ covariant form $\left(-\eta_{55}=\eta_{66}=1 ; \quad a, b, \cdots=0,1,2,3,5,6\right)$

$$
\left[M_{a b}, M_{c d}\right]=i\left(\eta_{b c} M_{a d}-\eta_{a c} M_{b d}-\eta_{b d} M_{a c}+\eta_{a d} M_{b c}\right)
$$

where $-\eta_{55}=\eta_{66}=1 ; \quad a, b, \cdots=0,1,2,3,5,6$. Spinor representation of $S U(2,2)$ can be written in terms of four-dimensional gamma matrices $\gamma_{\mu}$ that satisfy $\left(\left\{\gamma_{\mu}, \gamma_{\nu}\right\}=2 \eta_{\mu \nu}\right)$ and $\gamma_{5}=\gamma_{0} \gamma_{1} \gamma_{2} \gamma_{3}$ as follows:

$$
\Sigma_{\mu \nu}:=\frac{i}{4}\left[\gamma_{\mu}, \gamma_{\nu}\right] \quad \Sigma_{\mu 5}:=\frac{i}{2} \gamma_{\mu} \gamma_{5} \quad \Sigma_{\mu 6}:=\frac{1}{2} \gamma_{\mu} \quad \Sigma_{56}:=\frac{1}{2} \gamma_{5}
$$

We shall adopt the gamma matrix conventions of [43]

$$
\gamma^{0}=\gamma_{0}=\left(\begin{array}{cc}
\mathbf{1} & 0 \\
0 & -\mathbf{1}
\end{array}\right) \quad \gamma^{m}=-\gamma_{m}=\left(\begin{array}{cc}
0 & \sigma^{m} \\
-\sigma^{m} & 0
\end{array}\right) \Rightarrow \gamma_{5}=i\left(\begin{array}{ll}
0 & \mathbf{1} \\
\mathbf{1} & 0
\end{array}\right)
$$

where $\sigma^{m}, m=1,2,3$ are the usual Pauli matrices .

The oscillator construction of the positive energy unitary representations of the conformal group is simplest in the compact basis that is covariant with respect to its maximal compact subgroup, which is manifestly unitary. The maximal compact subgroup $S U(2)_{L} \times S U(2)_{R} \times U(1)_{E}$ is generated by the following generators

$$
\begin{aligned}
& L_{m}=\frac{1}{2}\left(\frac{1}{2} \varepsilon_{m n l} M_{n l}+M_{m 5}\right) \quad \longrightarrow S U(2)_{L} \\
& R_{m}=\frac{1}{2}\left(\frac{1}{2} \varepsilon_{m n l} M_{n l}-M_{m 5}\right) \quad \longrightarrow S U(2)_{R}
\end{aligned}
$$

where $m, n=1,2,3$. They satisfy

$$
\begin{aligned}
{\left[L_{m}, L_{n}\right] } & =i \varepsilon_{m n l} L_{l} \\
{\left[R_{m}, R_{n}\right] } & =i \varepsilon_{m n l} R_{l}
\end{aligned}
$$




$$
\left[L_{m}, R_{n}\right]=\left[E, L_{n}\right]=\left[E, R_{n}\right]=0 .
$$

The $U(1)_{E}$ generator $E=\frac{1}{2}\left(P_{0}+K_{0}\right)$ determines the three-grading and is simply the conformal Hamiltonian, whose spectrum is bounded from below in a positive energy representation. Denoting the maximal compact Lie subalgebra of $S U(2)_{L} \times S U(2)_{R} \times U(1)_{E}$ as $\mathcal{L}^{0}$, the conformal algebra $g$ has a three-graded decomposition

$$
g=\mathcal{L}^{+} \oplus \mathcal{L}^{0} \oplus \mathcal{L}^{-}
$$

where

$$
\left[\mathcal{L}^{0}, \mathcal{L}^{ \pm}\right]=\mathcal{L}^{ \pm}, \quad\left[E, \mathcal{L}^{ \pm}\right]= \pm \mathcal{L}^{ \pm}
$$

and the other commutators are equal to zero.

To construct positive energy unitary representations in a manifestly unitary way it is most convenient to work in the compact $S U(2)_{L} \times S U(2)_{R} \times U(1)_{E}$ covariant basis. The positive energy lowest weight UIRs of $S U(2,2)$ can then be constructed very simply by using the oscillator method of [59, 60, 3, 45].

Consider now $P$ copies ("generations" or "colors") of bosonic oscillators $a^{i}(R)=a_{i}(R)^{\dagger}$, $b^{j}(R)=b_{j}(R)^{\dagger}$ that satisfy

$$
\left[a_{i}(R), a^{j}(S)\right]=\delta_{i}^{j} \delta_{R S}, \quad\left[b_{i}(R), b^{j}(S)\right]=\delta_{i}^{j} \delta_{R S}
$$

with $i, j=1,2 ; R, S=1, \ldots, P$, We form $P$ sets of spinors $\Psi(R)$

$$
\Psi(R):=\left(\begin{array}{c}
a_{1}(R) \\
a_{2}(R) \\
b^{1}(R) \\
b^{2}(R)
\end{array}\right)
$$

whose Dirac conjugates are

$$
\bar{\Psi}(R) \equiv \Psi^{\dagger}(R) \gamma^{0}=\left(a^{1}(R), a^{2}(R),-b_{1}(R),-b_{2}(R)\right) .
$$

Then the bilinears

$$
\overline{\mathbf{\Psi}} \Sigma\left(M_{a b}\right) \Psi:=\sum_{R=1}^{P} \bar{\Psi}(R) \Sigma\left(M_{a b}\right) \Psi(R),
$$

realize the Lie algebra of $S U(2,2)$

$$
\left[\overline{\mathbf{\Psi}} \Sigma\left(M_{a b}\right) \mathbf{\Psi}, \overline{\mathbf{\Psi}} \Sigma\left(M_{c d}\right) \boldsymbol{\Psi}\right]=\overline{\mathbf{\Psi}}\left[\Sigma\left(M_{a b}\right), \Sigma\left(M_{c d}\right)\right] \boldsymbol{\Psi},
$$

The Fock space of the bosonic oscillators decompose into an infinite number of irreducible positive energy unitary representations of $S U(2,2)$ that belong to the holomorphic discrete 
series. In the above realization of $S U(2,2)$ non-compact generators are given by linear combinations of di-creation and di-annihilation operators of the form $\mathbf{a}^{i} \cdot \mathbf{b}^{j}$ and $\mathbf{a}_{i} \cdot \mathbf{b}_{j}{ }^{18}$. The generators, $L_{m}$, of $S U(2)_{L}$

$$
L_{i}^{k}:=\mathbf{a}^{k} \cdot \mathbf{a}_{i}-\frac{1}{2} \delta_{i}^{k} N_{a},
$$

whereas the generators $R_{m}$ of $S U(2)_{R}$ are

$$
R_{j}^{i}:=\mathbf{b}^{i} \cdot \mathbf{b}_{j}-\frac{1}{2} \delta_{j}^{i} N_{b}
$$

and the conformal hamiltonian $E$ is simply

$$
E=\frac{1}{2}\left(N_{a}+N_{b}+2 P\right)
$$

where $N_{a} \equiv \mathbf{a}^{i} \cdot \mathbf{a}_{i}, N_{b} \equiv \mathbf{b}^{j} \cdot \mathbf{b}_{j}$ are the bosonic number operators.

First step in constructing a positive energy UIR is to identify a set of states in the Fock space transforming in an irreducible representation $\left|\Omega\left(j_{L}, j_{R}, E\right)\right\rangle$ of $S U(2)_{L} \times S U(2)_{R} \times$ $U(1)_{E}$ where $\left(j_{L}, j_{R}\right)$ are $S U(2)_{L} \times S U(2)_{R}$ spins that are annihilated by all the operators $\mathbf{a}_{i} \cdot \mathbf{b}_{j}$ of subspace $\mathcal{L}^{-}$:

$$
\mathbf{a}_{i} \cdot \mathbf{b}_{j}\left|\Omega\left(j_{L}, j_{R}, E\right)\right\rangle=0 \quad \forall \mathbf{a}_{i} \cdot \mathbf{b}_{j} \in \mathcal{L}^{-}
$$

Acting repeatedly with the di-creation operators $\mathbf{a}^{i} \cdot \mathbf{b}^{j}$ of $\mathcal{L}^{+}$on $|\Omega\rangle$, one generates the basis of a positive energy UIR of the full group $S U(2,2)$ :

$$
\left|\Omega\left(j_{L}, j_{R}, E\right)\right\rangle, \mathcal{L}^{+}\left|\Omega\left(j_{L}, j_{R}, E\right)\right\rangle, \mathcal{L}^{+} \mathcal{L}^{+}\left|\Omega\left(j_{L}, j_{R}, E\right)\right\rangle, \cdots
$$

We shall refer to $\left|\Omega\left(j_{L}, j_{R}, E\right)\right\rangle$ as the lowest energy irreducible representation of $S U(2) \times$ $S U(2) \times U(1)$.

\section{B Six-dimensional $\Gamma$ matrices}

Our choice of $\Gamma$-matrices is given below:

$$
\begin{aligned}
& \Gamma_{0}=\sigma_{3} \otimes I_{2} \otimes I_{2}, \\
& \Gamma_{1}=i \sigma_{1} \otimes \sigma_{2} \otimes I_{2}, \\
& \Gamma_{2}=i \sigma_{1} \otimes \sigma_{1} \otimes \sigma_{2}, \\
& \Gamma_{3}=i \sigma_{1} \otimes \sigma_{3} \otimes \sigma_{2}, \\
& \Gamma_{4}=i \sigma_{2} \otimes I_{2} \otimes \sigma_{2}, \\
& \Gamma_{5}=i \sigma_{2} \otimes \sigma_{2} \otimes \sigma_{1},
\end{aligned}
$$

\footnotetext{
${ }^{18}$ The dot product $\cdot$ denotes summation over the generation or color index $R$, i.e. $\mathbf{a}^{i} \cdot \mathbf{b}^{j} \equiv$ $\sum_{R=1}^{P} a^{i}(R) b^{j}(R)$, etc.
} 
where $\sigma_{1}, \sigma_{2}$ and $\sigma_{3}$ are the Pauli matrices and $I_{2}$ is the $2 \times 2$ identity matrix.

Therefore,

$$
\Gamma_{7}=-\Gamma_{0} \Gamma_{1} \Gamma_{2} \Gamma_{3} \Gamma_{4} \Gamma_{5}=-\sigma_{2} \otimes \sigma_{2} \otimes \sigma_{3} .
$$

The $\Sigma$ matrices in $d=6$, the analogs of Pauli matrices $\sigma_{\mu}$ in $d=4$, are given below.

$$
\begin{aligned}
& \left(\Sigma_{0}\right)=-i \sigma_{2} \otimes \sigma_{3}, \\
& \left(\Sigma_{1}\right)=i \sigma_{2} \otimes I_{2}, \\
& \left(\Sigma_{2}\right)=i \sigma_{1} \otimes \sigma_{2}, \\
& \left(\Sigma_{3}\right)=i \sigma_{3} \otimes \sigma_{2}, \\
& \left(\Sigma_{4}\right)=I_{2} \otimes \sigma_{2}, \\
& \left(\Sigma_{5}\right)=\sigma_{2} \otimes \sigma_{1}
\end{aligned}
$$

\section{References}

[1] J. M. Maldacena, "The Large N limit of superconformal field theories and supergravity," Adv. Theor. Math. Phys. 2, 231-252 (1998). [hep-th/9711200].

[2] E. Witten, "Anti-de Sitter space and holography," Adv. Theor. Math. Phys. 2, 253291 (1998). [hep-th/9802150].

[3] M. Gunaydin, N. Marcus, "The Spectrum of the $S^{5}$ Compactification of the Chiral $N=2, D=10$ Supergravity and the Unitary Supermultiplets of $U(2,2 / 4)$," Class. Quant. Grav. 2, L11 (1985).

[4] M. Günaydin, P. van Nieuwenhuizen and N. P. Warner, "General construction of the unitary representations of anti-de Sitter superalgebras and the spectrum of the $S^{4}$ compactification of eleven dimensional supergravity," Nucl. Phys. B 255, 63 (1985).

[5] M. Gunaydin and N. P. Warner, "Unitary supermultiplets of $O S p(8 / 4, \mathbb{R})$ and the spectrum of the $S^{7}$ compactification of eleven-dimensional supergravity," Nucl. Phys. B 272, 99 (1986). M. Gunaydin and S. J. Hyun, "Unitary lowest weight representations of the noncompact supergroup $O S p(2 n / 2 m, \mathbb{R})$," J. Math. Phys. 29, 2367 (1988).

[6] P. A. M. Dirac, J. Math. Phys. 4 (1963) 901; see also the prescient paper by E. Majorana, Nuovo Cimento 9 (1932) 335.

[7] C. Fronsdal, Phys. Rev. D26 (1982) 1988; M. Flato and C. Fronsdal, J. Math. Phys. 22 (1981) 1100; E. Angelopoulos, M. Flato, C. Fronsdal and D. Steinheimer, Phys. Rev. D23 (1981) 1278. 
[8] S. Fernando and M. Gunaydin, "Minimal unitary representation of SU(2,2) and its deformations as massless conformal fields and their supersymmetric extensions," arXiv:0908.3624 [hep-th].

[9] S. Fernando, M. Gunaydin, "Minimal unitary representation of $S O^{*}(8)=S O(6,2)$ and its $S U(2)$ deformations as massless $6 \mathrm{D}$ conformal fields and their supersymmetric extensions," Nucl. Phys. B841, 339-387 (2010). [arXiv:1005.3580 [hep-th]]; "SU(2) deformations of the minimal unitary representation of $O S p\left(8^{*} \mid 2 N\right)$ as massless $6 \mathrm{D}$ conformal supermultiplets," Nucl. Phys. B843, 784-815 (2011). [arXiv:1008.0702 [hep-th]].

[10] Z. Bern, L. J. Dixon, R. Roiban, "Is N = 8 supergravity ultraviolet finite?," Phys. Lett. B644, 265-271 (2007). [hep-th/0611086].

[11] Z. Bern, J. J. Carrasco, L. J. Dixon, H. Johansson, D. A. Kosower, R. Roiban, "Three-Loop Superfiniteness of N=8 Supergravity," Phys. Rev. Lett. 98, 161303 (2007). [hep-th/0702112].

[12] Z. Bern, J. J. M. Carrasco, L. J. Dixon, H. Johansson, R. Roiban, "Manifest Ultraviolet Behavior for the Three-Loop Four-Point Amplitude of N=8 Supergravity," Phys. Rev. D78, 105019 (2008). [arXiv:0808.4112 [hep-th]].

[13] Z. Bern, J. J. Carrasco, L. J. Dixon, H. Johansson, R. Roiban, "The Ultraviolet Behavior of N=8 Supergravity at Four Loops," Phys. Rev. Lett. 103, 081301 (2009). [arXiv:0905.2326 [hep-th]].

[14] Z. Bern, J. J. Carrasco, L. J. Dixon, H. Johansson, R. Roiban, "Amplitudes and Ultraviolet Behavior of N=8 Supergravity," [arXiv:1103.1848 [hep-th]].

[15] Z. Bern, L. J. Dixon, M. Perelstein and J. S. Rozowsky, "Multi-leg one-loop gravity amplitudes from gauge theory," Nucl. Phys. B 546, 423 (1999) [hep-th/9811140].

[16] Z. Bern, N. E. J. Bjerrum-Bohr and D. C. Dunbar, "Inherited twistor-space structure of gravity loop amplitudes," JHEP 0505, 056 (2005) [hep-th/0501137].

[17] N. E. J. Bjerrum-Bohr, D. C. Dunbar and H. Ita, "Six-point one-loop N = 8 supergravity NMHV amplitudes and their IR behaviour," Phys. Lett. B 621, 183 (2005) [hep-th/0503102]];

N. E. J. Bjerrum-Bohr, D. C. Dunbar, H. Ita, W. B. Perkins and K. Risager, "The no-triangle hypothesis for $\mathrm{N}=8$ supergravity," JHEP 0612, 072 (2006) [hepth/0610043].

[18] N. E. J. Bjerrum-Bohr, P. Vanhove, JHEP 0804, 065 (2008). [arXiv:0802.0868 [hepth]]. 
[19] R. Kallosh, "The Effective Action of N=8 Supergravity," [arXiv:0711.2108 [hep-th]].

[20] G. Chalmers, "On the finiteness of $N=8$ quantum supergravity," hep-th/0008162;

M. B. Green, J. G. Russo and P. Vanhove, "Non-renormalisation conditions in type II string theory and maximal supergravity," JHEP 0702, 099 (2007) [hepth/0610299v4].

[21] M. B. Green, H. Ooguri, J. H. Schwarz, "Nondecoupling of Maximal Supergravity from the Superstring," Phys. Rev. Lett. 99, 041601 (2007). [arXiv:0704.0777 [hepth]].

[22] M. B. Green, J. G. Russo, P. Vanhove, "Automorphic properties of low energy string amplitudes in various dimensions," Phys. Rev. D81, 086008 (2010). [arXiv:1001.2535 [hep-th]].

[23] M. B. Green, J. G. Russo, P. Vanhove, "String theory dualities and supergravity divergences," JHEP 1006, 075 (2010). [arXiv:1002.3805 [hep-th]].

[24] R. Kallosh, "N=8 Counterterms and $E_{7(7)}$ Current Conservation," JHEP 1106, 073 (2011) [arXiv:1104.5480 [hep-th]].

[25] R. Kallosh, " $E_{7(7)}$ Symmetry and Finiteness of N=8 Supergravity," [arXiv:1103.4115 [hep-th]].

[26] G. Bossard, H. Nicolai, "Counterterms vs. Dualities," [arXiv:1105.1273 [hep-th]].

[27] R. Kallosh, "N=8 Supergravity on the Light Cone," Phys. Rev. D80, 105022 (2009). [arXiv:0903.4630 [hep-th]].

[28] M. Gunaydin, N. Marcus, "The Unitary Supermultiplet Of $N=8$ Conformal Superalgebra Involving Fields Of Spin $\leq$ 2," Class. Quant. Grav. 2, L19 (1985).

[29] J. M. Drummond, P. J. Heslop, P. S. Howe, "A Note on N=8 counterterms," [arXiv:1008.4939 [hep-th]].

[30] N. Beisert, H. Elvang, D. Z. Freedman, M. Kiermaier, A. Morales, S. Stieberger, " $E_{7(7)}$ constraints on counterterms in $N=8$ supergravity," Phys. Lett. B694, 265271 (2010). [arXiv:1009.1643 [hep-th]].

[31] L. Brink, P. S. Howe, "The N=8 Supergravity In Superspace," Phys. Lett. B88, 268 (1979).

[32] R. E. Kallosh, "Counterterms in extended supergravities," Phys. Lett. B99, 122-127 (1981). 
[33] P. S. Howe, U. Lindstrom, "Higher Order Invariants In Extended Supergravity," Nucl. Phys. B181, 487 (1981).

[34] J. M. Drummond, P. J. Heslop, P. S. Howe, S. F. Kerstan, "Integral invariants in $\mathrm{N}=4 \mathrm{SYM}$ and the effective action for coincident D-branes," JHEP 0308, 016 (2003). [hep-th/0305202].

[35] J. Broedel, L. J. Dixon, " $R^{4}$ counterterm and $E_{7(7)}$ symmetry in maximal supergravity," JHEP 1005, 003 (2010). [arXiv:0911.5704 [hep-th]].

[36] H. Elvang, M. Kiermaier, "Stringy KLT relations, global symmetries, and $E_{7(7)}$ violation," JHEP 1010, 108 (2010). [arXiv:1007.4813 [hep-th]].

[37] G. Bossard, P. S. Howe, K. S. Stelle, "On duality symmetries of supergravity invariants," JHEP 1101, 020 (2011). [arXiv:1009.0743 [hep-th]]

[38] E. Witten, "Some comments on string dynamics," [hep-th/9507121].

[39] A. Strominger, "Open p-branes," Phys. Lett. B 383, 44 (1996) [arXiv:hepth/9512059].

[40] C. M. Hull, "Strongly coupled gravity and duality," Nucl. Phys. B 583, 237 (2000) [arXiv:hep-th/0004195].

[41] C. M. Hull, "Symmetries and compactifications of $(4,0)$ conformal gravity," JHEP 0012, 007 (2000) [arXiv:hep-th/0011215].

[42] G. Mack and A. Salam, "Finite component field representations of the conformal group," Annals Phys. 53, 174 (1969); G. Mack, "All unitary ray representations of the conformal group $S U(2,2)$ with positive energy," Commun. Math. Phys. 55, 1 (1977).

[43] M. Gunaydin, D. Minic, M. Zagermann, "Novel supermultiplets of $S U(2,2 \mid 4)$ and the $A d S_{5} / C F T_{4}$ duality," Nucl. Phys. B544, 737-758 (1999). [hep-th/9810226].

[44] B. Binegar, "Relativistic Field Theories In Three-dimensions," J. Math. Phys. 23, 1511 (1982).

[45] M. Gunaydin, D. Minic, M. Zagermann, " $4 D$ doubleton conformal theories, CPT and IIB string on $A d S_{5} \times S^{5}$," Nucl. Phys. B534, 96-120 (1998). [hep-th/9806042].

[46] A. O. Barut and H. Kleinert, "Dynamical Group $O(4,2)$ for Baryons and the Behaviour of Form factors," Phys. Rev. 1611464 (1967); "Current Operators and Majorana Equation for the Hydrogen Atom from Dynamical Groups," Phys. Rev. 157, 1180-1183 (1967). 
[47] G. Mack and I. Todorov, "Irreducibility of the ladder representations of $U(2,2)$ when restricted to the Poincare subgroup," J. Math. Phys. 10 (1969) 2078-2085.

[48] W. Siegel, "On-shell O(n) Supergravity In Superspace," Nucl. Phys. B177, 325 (1981).

[49] P. S. Howe, K. S. Stelle, P. K. Townsend, "Supercurrents," Nucl. Phys. B192, 332 (1981).

[50] S. Ferrara, E. Sokatchev, "Superconformal interpretation of BPS states in AdS geometries," Int. J. Theor. Phys. 40, 935-984 (2001). [hep-th/0005151].

[51] M. Gunaydin, S. J. Hyun, "Unitary Lowest Weight Representations of the Noncompact Supergroup $O S p(2 n / 2 m, \mathbb{R})$," J. Math. Phys. 29, 2367 (1988).

[52] N. Marcus, J. H. Schwarz, "Three-Dimensional Supergravity Theories," Nucl. Phys. B228, 145 (1983).

[53] H. Nicolai, H. Samtleben, "Maximal gauged supergravity in three-dimensions," Phys. Rev. Lett. 86, 1686-1689 (2001). [hep-th/0010076].

[54] H. Samtleben, R. Wimmer, " $N=8$ Superspace Constraints for Three-dimensional Gauge Theories," JHEP 1002, 070 (2010). [arXiv:0912.1358 [hep-th]].

[55] M. Günaydin and S. Takemae, "Unitary supermultiplets of $O S p\left(8^{*} \mid 4\right)$ and the $A d S_{7} / C F T_{6}$ duality," Nucl. Phys. B 578, 405 (2000) [hep-th/9910110].

[56] S. Fernando, M. Gunaydin, S. Takemae, "Supercoherent states of $O S p\left(8^{*} \mid 2 N\right)$, conformal superfields and the $A d S_{7} / C F T_{6}$ duality," Nucl. Phys. B628, 79-111 (2002). [hep-th/0106161].

[57] J. Greitz, P. S. Howe, "Maximal supergravity in three dimensions: supergeometry and differential forms," JHEP 1107, 071 (2011). [arXiv:1103.2730 [hep-th]].

[58] S. Ferrara, E. Sokatchev, "Representations of superconformal algebras in the $\operatorname{AdS}(7 / 4)$ / CFT(6/3) correspondence," J. Math. Phys. 42, 3015-3026 (2001). [hepth/0010117].

[59] M. Günaydin and C. Saclioglu, "Oscillator like unitary representations of noncompact groups with a Jordan structure and the non-compact groups of supergravity," Commun. Math. Phys. 87, 159 (1982).

[60] I. Bars and M. Günaydin, "Unitary representations of non-compact supergroups," Commun. Math. Phys. 91, 31 (1983); 
[61] M. Günaydin and R. J. Scalise, "Unitary lowest weight representations of the noncompact supergroup $O S p\left(2 m^{*} / 2 n\right)$," J. Math. Phys. 32, 599 (1991).

[62] M. Günaydin, "Generalized conformal and superconformal group actions and Jordan algebras," Mod. Phys. Lett. A 8, 1407 (1993) [hep-th/9301050]. M. Günaydin, in "Elementary particles and the universe: Essays in honor of Murray Gell-Mann", ed. by J.H. Schwarz, Cambridge Univ. Press (1991) pp 99-119.

[63] C. Cheung, D. O'Connell, "Amplitudes and Spinor-Helicity in Six Dimensions," JHEP 0907, 075 (2009). [arXiv:0902.0981 [hep-th]].

[64] T. Dennen, Y. -t. Huang, W. Siegel, "Supertwistor space for 6D maximal super Yang-Mills," JHEP 1004, 127 (2010). [arXiv:0910.2688 [hep-th]].

[65] P. Claus, R. Kallosh and A. Van Proeyen, "M 5-brane and superconformal (0,2) tensor multiplet in 6 dimensions," Nucl. Phys. B 518, 117 (1998) [hep-th/9711161].

[66] M. Günaydin and R. J. Scalise, "Unitary lowest weight representations of the noncompact supergroup $O S p\left(2 m^{*} / 2 n\right)$," J. Math. Phys. 32, 599 (1991).

[67] P. S. Howe, G. Sierra, P. K. Townsend, "Supersymmetry in Six-Dimensions," Nucl. Phys. B221, 331 (1983).

[68] S. Ferrara and E. Sokatchev, "Representations of $(1,0)$ and $(2,0)$ superconformal algebras in six dimensions: Massless and short superfields," Lett. Math. Phys. 51, 55 (2000) [hep-th/0001178];

[69] P. Arvidsson, E. Flink and M. Henningson, "Supersymmetric coupling of a selfdual string to a $(2,0)$ tensor multiplet background," JHEP 0311, 015 (2003) [arXiv:hepth/0309244].

[70] J. M. F. Labastida and T. R. Morris, "Massless Mixed Symmetry Bosonic Free Fields," Phys. Lett. B 180, 101 (1986).

[71] A. Campoleoni, D. Francia, J. Mourad and A. Sagnotti, "Unconstrained Higher Spins of Mixed Symmetry. I. Bose Fields," Nucl. Phys. B 815, 289 (2009) [arXiv:0810.4350 [hep-th]].

[72] E. Witten, "New 'gauge' theories in six-dimensions," JHEP 9801, 001 (1998) [Adv. Theor. Math. Phys. 2, 61 (1998)] [hep-th/9710065].

[73] D. Gaiotto, "N=2 dualities," arXiv:0904.2715 [hep-th].

[74] D. Gaiotto and J. Maldacena, "The Gravity duals of $\mathrm{N}=2$ superconformal field theories," arXiv:0904.4466 [hep-th]. 
[75] E. Witten, "Perturbative gauge theory as a string theory in twistor space," Commun. Math. Phys. 252, 189 (2004) [hep-th/0312171].

[76] N. Berkovits, "An Alternative string theory in twistor space for $\mathrm{N}=4$ superYangMills," Phys. Rev. Lett. 93, 011601 (2004) [hep-th/0402045].

[77] N. Berkovits and E. Witten, "Conformal supergravity in twistor-string theory," JHEP 0408, 009 (2004) [hep-th/0406051].

[78] J. Broedel and B. Wurm, "New Twistor String Theories revisited," Phys. Lett. B 675, 463 (2009) [arXiv:0902.0550 [hep-th]].

[79] S. Weinberg, "Photons and Gravitons in s Matrix Theory: Derivation of Charge Conservation and Equality of Gravitational and Inertial Mass," Phys. Rev. 135, B1049 (1964).

[80] S. R. Coleman and J. Mandula, "All Possible Symmetries Of The S Matrix," Phys. Rev. 159, 1251 (1967).

[81] A. Sagnotti and M. Taronna, "String Lessons for Higher-Spin Interactions," Nucl. Phys. B 842, 299 (2011) [arXiv:1006.5242 [hep-th]].

[82] M. Taronna, "Higher Spins and String Interactions," arXiv:1005.3061 [hep-th].

[83] S. Weinberg and E. Witten, "Limits on Massless Particles," Phys. Lett. B 96, 59 (1980).

[84] M. Porrati, "Universal Limits on Massless High-Spin Particles," Phys. Rev. D 78, 065016 (2008) [arXiv:0804.4672 [hep-th]].

[85] R. Haag, J. T. Lopuszanski and M. Sohnius, "All Possible Generators of Supersymmetries of the s Matrix," Nucl. Phys. B 88, 257 (1975).

[86] A. K. H. Bengtsson, I. Bengtsson and L. Brink, "Cubic Interaction Terms For Arbitrarily Extended Supermultiplets," Nucl. Phys. B 227, 41 (1983).

[87] A. K. H. Bengtsson, I. Bengtsson and L. Brink, "Cubic Interaction Terms For Arbitrary Spin," Nucl. Phys. B 227, 31 (1983).

[88] R. R. Metsaev, "Cubic interaction vertices of totally symmetric and mixed symmetry massless representations of the Poincare group in $\mathrm{D}=6$ space-time," Phys. Lett. B 309, 39 (1993).

[89] R. R. Metsaev, "Cubic interaction vertices for fermionic and bosonic arbitrary spin fields," Nucl. Phys. B 859, 13 (2012) [arXiv:0712.3526 [hep-th]]. 
[90] F. A. Berends, G. J. H. Burgers and H. van Dam, "On The Theoretical Problems In Constructing Interactions Involving Higher Spin Massless Particles," Nucl. Phys. B 260, 295 (1985).

[91] R. Manvelyan, K. Mkrtchyan and W. Ruhl, "General trilinear interaction for arbitrary even higher spin gauge fields," Nucl. Phys. B 836, 204 (2010) [arXiv:1003.2877 [hep-th]].

[92] W. Ruehl, "Solving Noether's equations for gauge invariant local Lagrangians of N arbitrary higher even spin fields," arXiv:1108.0225 [hep-th].

[93] X. Bekaert, N. Boulanger and P. Sundell, "How higher-spin gravity surpasses the spin two barrier: no-go theorems versus yes-go examples," arXiv:1007.0435 [hep-th].

[94] Y. -t. Huang and A. E. Lipstein, "Amplitudes of 3D and 6D Maximal Superconformal Theories in Supertwistor Space," JHEP 1010, 007 (2010) [arXiv:1004.4735 [hep-th]].

[95] B. Czech, Y. -t. Huang and M. Rozali, "Amplitudes for Multiple M5 Branes," arXiv:1110.2791 [hep-th].

[96] Y. Tanii, "N=8 Supergravity In Six-dimensions," Phys. Lett. B145, 197-200 (1984).

[97] M. R. Douglas, "On D=5 super Yang-Mills theory and (2,0) theory," JHEP 1102, 011 (2011) [arXiv:1012.2880 [hep-th]].

[98] N. Lambert, C. Papageorgakis, M. Schmidt-Sommerfeld, "M5-Branes, D4-Branes and Quantum 5D super-Yang-Mills," JHEP 1101, 083 (2011). [arXiv:1012.2882 [hep-th]].

[99] H. Elvang, D. Z. Freedman and M. Kiermaier, "A simple approach to counterterms in N=8 supergravity," JHEP 1011, 016 (2010) [arXiv:1003.5018 [hep-th]].

[100] H. Elvang, D. Z. Freedman and M. Kiermaier, "SUSY Ward identities, Superamplitudes, and Counterterms," arXiv:1012.3401 [hep-th].

[101] D. Z. Freedman and E. Tonni, "The $D^{2 k} R^{4}$ Invariants of N=8 Supergravity," JHEP 1104, 006 (2011) [arXiv:1101.1672 [hep-th]].

[102] G. Bossard, P. S. Howe, K. S. Stelle and P. Vanhove, "The vanishing volume of $\mathrm{D}=4$ superspace," arXiv:1105.6087 [hep-th]. 